\title{
Contribución al conocimiento de los moluscos del Pleistoceno Inferior del yacimiento de Barranco León (Cuenca de Guadix-Baza)
}

\author{
Joaquin ALBESA* \& Fernando ROBLES
}

Departamento de Botánica y Geología. Facultad de Biología. Universitat de València. C/ Dr. Moliner, 50.46100 Burjassot. joaquin.albesa@uv.es

* Corresponding author

Albesa, J. \& Robles, F. 2020. Contribución al conocimiento de los moluscos del Pleistoceno Inferior del yacimiento de Barranco León (Cuenca de Guadix-Baza). [Contribution to the knowledge of the molluscs of the Lower Pleistocene of the Barranco León site (Guadix-Baza Basin)]. Spanish Journal of Palaeontology, 35 (1), 125-146.

Manuscript received 1 March 2019

Manuscript accepted 20 April 2020 https://doi.org/10.7203/sjp.35.1.17324

(C) Sociedad Española de Paleontología ISSN 2255-0550

\section{RESUMEN}

Se exponen los resultados obtenidos en el análisis de la asociación malacológica de dos muestras pertenecientes a la capa D del yacimiento del Pleistoceno inferior de Barranco León (Cuenca de Guadix-Baza). Los principales resultados obtenidos son la relación de especies que componen la asociación malacológica y su abundancia relativa, que indican su marcado carácter acuático. Por otra parte, pese a las limitaciones impuestas por la escasez de información tafonómica y la cautela que sugieren ciertos indicadores, se aporta, a partir del análisis de las exigencias ecológicas de cada taxón, una interpretación paleoambiental sobre las condiciones existentes en el momento del depósito. Éstas corresponderían a la zona marginal de un lago permanente, oligohalino, de régimen léntico, con escasa profundidad, presencia de macrófitos y existencia de surgencias termales. Este lago estaría rodeado por vegetación litoral en lo que constituiría una franja de carácter higrófilo y se encontraría enmarcado en un espacio abierto en el que las condiciones climáticas serían de carácter más xérico y en las que encontraríamos un matorral mediterráneo salpicado de agrupaciones de árboles.

Palabras clave: Moluscos continentales, Pleistoceno, Cuenca de Guadix-Baza, Barranco León.

\begin{abstract}
The results obtained in the analysis of the malacological assemblage of two samples belonging to layer D of the Lower Pleistocene deposit of Barranco León (Guadix-Baza Basin) are presented. The main results obtained are the list of species of the malacological assemblage and their relative abundance, which indicate their marked aquatic character. On the other hand, despite the limitations imposed by the scarcity of taphonomic information and the caution suggested by certain indicators, a paleoenvironmental interpretation of the conditions existing at the time of the deposit is provided, based on the analysis of the ecological requirements of each taxon. These would correspond to the marginal zone of a permanent lake, oligohaline, of lentic regime, with little depth, presence of macrophytes and existence of thermal upwellings. This lake would be surrounded by littoral vegetation in what would constitute a strip of hygrophilic flora framed in an open space in which the climatic conditions would be more xeric in character and in we would find a Mediterranean scrub otted with tree clusters.
\end{abstract}

Keywords: Continental molluscs, Pleistocene, Guadix-Baza Basin, Barranco León. 


\section{INTRODUCCIÓN}

En Europa son numerosos los yacimientos pleistocenos, así como los estudios sobre las asociaciones de moluscos continentales que contienen registradas que han posibilitado un elevado conocimiento regional (e.g. Esu \& Girotti, 1975, 1991; Schlickum \& Puissegur, 1977, 1978; Meijer, 1990; Gliozzi et al., 1997; Benvenutti, 1998; Petronio et al., 2000-2002; Esu \& Ghinassi, 2013). Recientemente, Georgopoulou et al. (2016) estudiaron la distribución biogeográfica y evolución de la diversidad durante el Cuaternario y observaron que, una vez finalizado el Plioceno se produjo a lo largo del Pleistoceno una progresiva pérdida de diversidad en relación con la desaparición de lagos de larga vida y la disponibilidad de masas de agua dulce. Pese a la disminución generalizada iniciada, la existencia de lagos antiguos en varias regiones europeas durante el Gelasiense (e.g. Bresse, East Anglian Crag, Kos y Tiberino) y la persistencia de algunos de éstos en Francia e Italia durante el Calabriense (e.g. Bresse y Tiberino) con la consiguiente estabilidad ambiental que ello suponía, se mantuvo la diversidad en dichas cuencas. En contraste, durante el Pleistoceno inferior en la Península Ibérica sólo persistieron pequeñas masas de agua con una fauna menos diversa.

La Cuenca de Guadix-Baza constituye uno de esos cuerpos menores existentes en el Calabriense de la Península Ibérica en el que, pese a la existencia de un elevado número de yacimientos en los que los moluscos exhiben un buen grado de preservación, los trabajos previos se reducen a los de Anadón et al. (1987), Robles (1989), Anadón et al. (2003) y De Marfà (2007). El primero y los dos últimos aportan datos concretos sobre el propio yacimiento de Barranco León: Anadón et al. (1987) ofrecen datos de dos perfiles que contienen respectivamente los yacimientos clásicos de Barranco León 1 y de Barranco León 2 y 3 y Anadón et al. (2003) y De Marfà (2007) aportan datos de la capa D. Por otro lado, Robles (1989) suministra información sobre las asociaciones de moluscos de diversos yacimientos de la cuenca entre los que no se encuentra el que nos ocupa.

Por lo que se refiere a los trabajos sobre moluscos actuales, cuyo conocimiento ha resultado de utilidad en la determinación de los taxones estudiados a la vez que ha contribuido a discutir aspectos biogeográficos e inferir las condiciones paleoecológicas, son destacables los de Alonso (1975a, 1975b), Arconada \& Ramos (2001, 2003, 2006), Arconada et al. (2007a) y Delicado et al. (2012). Alonso (1975a, 1975b) suministran abundantes datos sobre la fauna que caracteriza la depresión de Granada, y Arconada \& Ramos (2001, 2003, 2006), Arconada et al. (2007a) y Delicado et al. (2012) hacen referencia a los moluscos prosobranquios de algunas de las fuentes, tanto próximas a la zona que nos ocupa como de otras situadas alrededor de ésta. Si bien no se refieren exactamente a la zona en la que se localiza el yacimiento de Barranco León, reseñables son también trabajos de zonas próximas que contienen especies afines a las estudiadas en esta publicación (Boeters, 2003; Arconada et al., 2007b) y de otras relativamente alejadas, pero con importante valor biogeográfico (Pérez-Quintero et al., 2004; Pérez-Quintero, 2007, 2009, 2011a, 2011b, 2012, 2013).

Por lo tanto, el presente trabajo persigue mejorar el conocimiento de los moluscos pleistocenos de la Península Ibérica y de la Cuenca de Guadix-Baza, con la consiguiente posibilidad de precisar en el futuro los patrones de distribución de los taxones y los valores de diversidad que actualmente se infieren para el Cuaternario de Europa, así como del yacimiento de Barranco León muy en particular. En ese sentido se exponen los resultados obtenidos en el análisis del contenido malacológico de dos muestras pertenecientes a la capa $\mathrm{D}$ del yacimiento pleistoceno de Barranco León. Se suministra un listado de taxones, discutiéndose su posición taxonómica, así como sus exigencias ecológicas y distribución, aportándose, así mismo, valores sobre la abundancia de cada taxón y realizándose, a partir de la consideración de todo ello, una inferencia ecológica de las condiciones reinantes en el momento del depósito. Esta interpretación ecológica complementa y apoya además los resultados obtenidos en estudios efectuados desde otras disciplinas por diversos autores.

\section{CONTEXTO GEOLÓGICO}

La Cuenca de Guadix-Baza es una depresión intramontañosa con dirección NE-SO localizada en el interior de la cordillera Bética que posee unos $110 \mathrm{~km}$ de longitud y que se encuentra dividida en las subcuencas de Guadix y de Baza, localizada la primera en la zona occidental y la segunda en la oriental (Fig. 1). La cuenca se encuentra rellena por sedimentos que comenzaron a depositarse en el Mioceno superior a través de una potente serie sedimentaria marina de unos $1000 \mathrm{~m}$. de espesor, cuyos principales afloramientos se encuentran en las zonas marginales de la cuenca (García-Aguilar et al., 2014). A techo de la secuencia, se disponen sedimentos continentales que abarcan una edad comprendida entre el Turoliense y el Pleistoceno superior en lo que constituye la secuencia continua de mayor espesor de la Península Ibérica (GarcíaAguilar \& Martín, 2000). Estos sedimentos se agrupan en dos bloques sedimentarios, el correspondiente al Turoliense y el que se refiere al Plio-Pleistoceno, siendo tres las formaciones litoestratigráficas que albergan, Guadix, Gorafe-Huélago y Baza (Vera, 1970).

El yacimiento de Barranco León se encuentra situado dos kilómetros al este de la población de Orce en la zona 


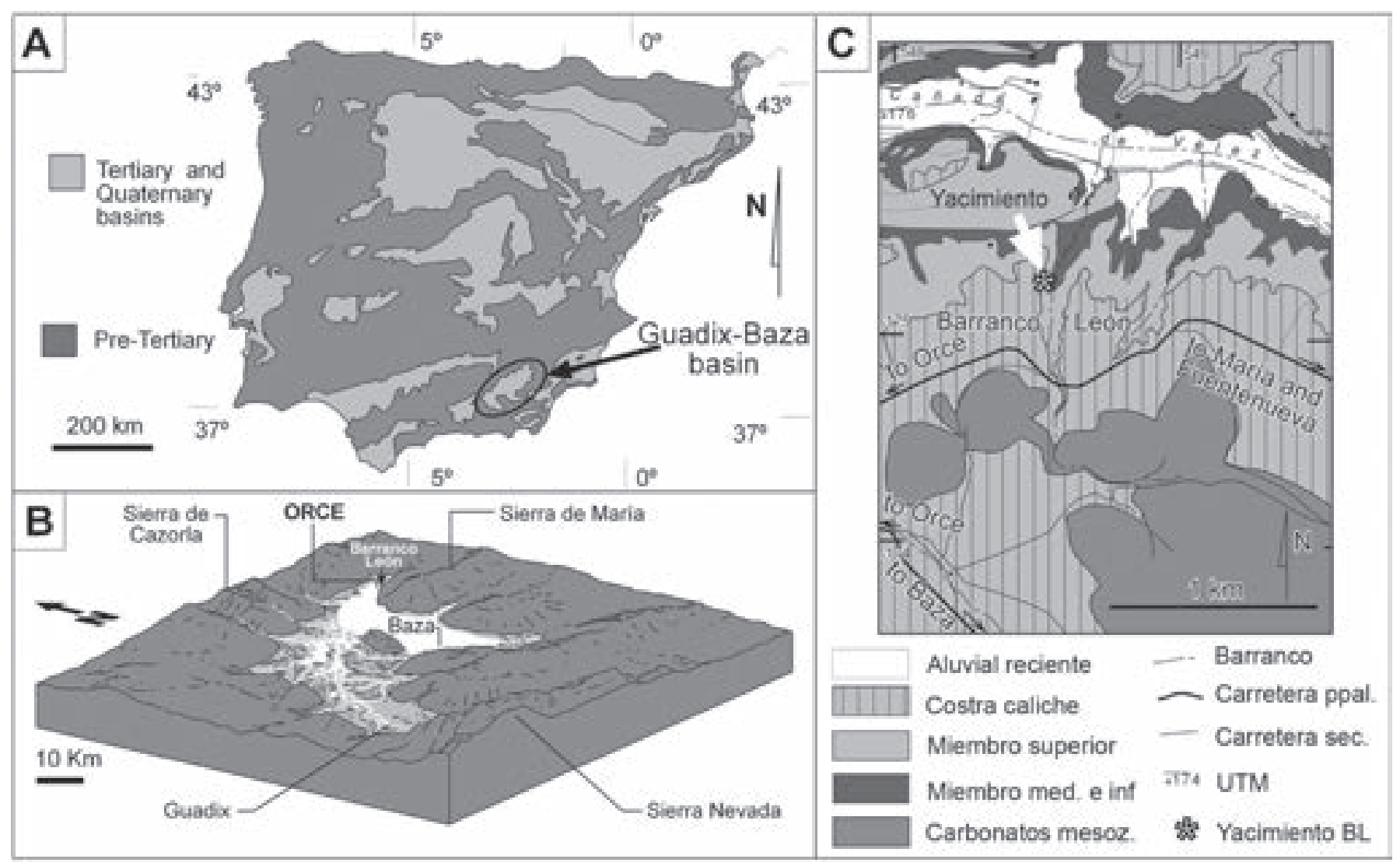

Figura 1. a) Localización de la Cuenca de Guadix-Baza. b) Reconstrucción paleogeográfica de la Cuenca de Guadix-Baza. c) Localización del yacimiento de Barranco León. Modificado de Anadón et al. (2014).

más oriental de la subcuenca de Baza, habiendo sido atribuido al Pleistoceno inferior y datado en alrededor de 1,4 Ma (Toro-Moyano et al., 2013). Dicho yacimiento se localiza en materiales pertenecientes a la $\mathrm{V}$ unidad lacustre, atribuida al Calabriense y conformada por una alternancia de margas, arenas y carbonatos. Las características de los carbonatos evidencian una intensa actividad hidrotermal (Medin et al., 2017). Los materiales que integran esta unidad fueron depositados, en términos generales, durante la fase de mayor expansión de un paleolago que ocupó la parte oriental de la Cuenca de Guadix-Baza en el Pleistoceno temprano (García-Aguilar \& Palmqvist, 2011), localizándose el yacimiento en sedimentos depositados cerca del margen del lago (Oms et al., 2011). Los sedimentos de la sección estudiada en la que se localiza el yacimiento (Fig. 2) indican un entorno pantanoso con excepción del propio yacimiento que muestra características fluviales (Duval et al., 2012). La capa que integra el yacimiento, que recibe la denominación actual de capa $\mathrm{D}$ y posee un espesor de $70 \mathrm{~cm}$ (Anadon et al., 2003), representa una fina secuencia de relleno de un paleocanal fluvial que erosionó sedimentos pantanosos depositados previamente (Arribas \& Palmqvist, 2002).

\section{MATERIAL Y MÉTODOS}

El material sobre el que se ha elaborado el estudio procede del levigado de sedimentos para la extracción de fósiles de micromamíferos en dos muestras pertenecientes a la capa D. Las muestras estudiadas, BL01 L63 de 2,1 g y BL02 J55 de 5,2 g, están integradas exclusivamente por moluscos, correspondiendo respectivamente a las campañas de 2001 y 2002. Las muestras fueron proporcionadas por personal del Instituto de Paleontología "Miquel Crusafont" de Sabadell, siendo depositadas definitivamente en el Museo de la Universitat de València de Historia Natural con los números MGUV-36609 a 36636.

Para determinar las distintas especies que contenía cada muestra se separaron, bajo lupa binocular, todos los ejemplares que parecían pertenecer a taxones distintos. Una vez separados se realizó la determinación a partir de su estudio con la propia lupa binocular y, en algunos casos, mediante el uso de un microscopio electrónico de barrido modelo Hitachi S-4100. La ordenación taxonómica se realizó según MolluscaBase (2018).

Para evaluar la abundancia de cada taxón en las distintas muestras, se dividió cada una de ellas en submuestras alícuotas de alrededor de 80 ejemplares para, a continuación, efectuar el conteo sobre cinco de éstas 


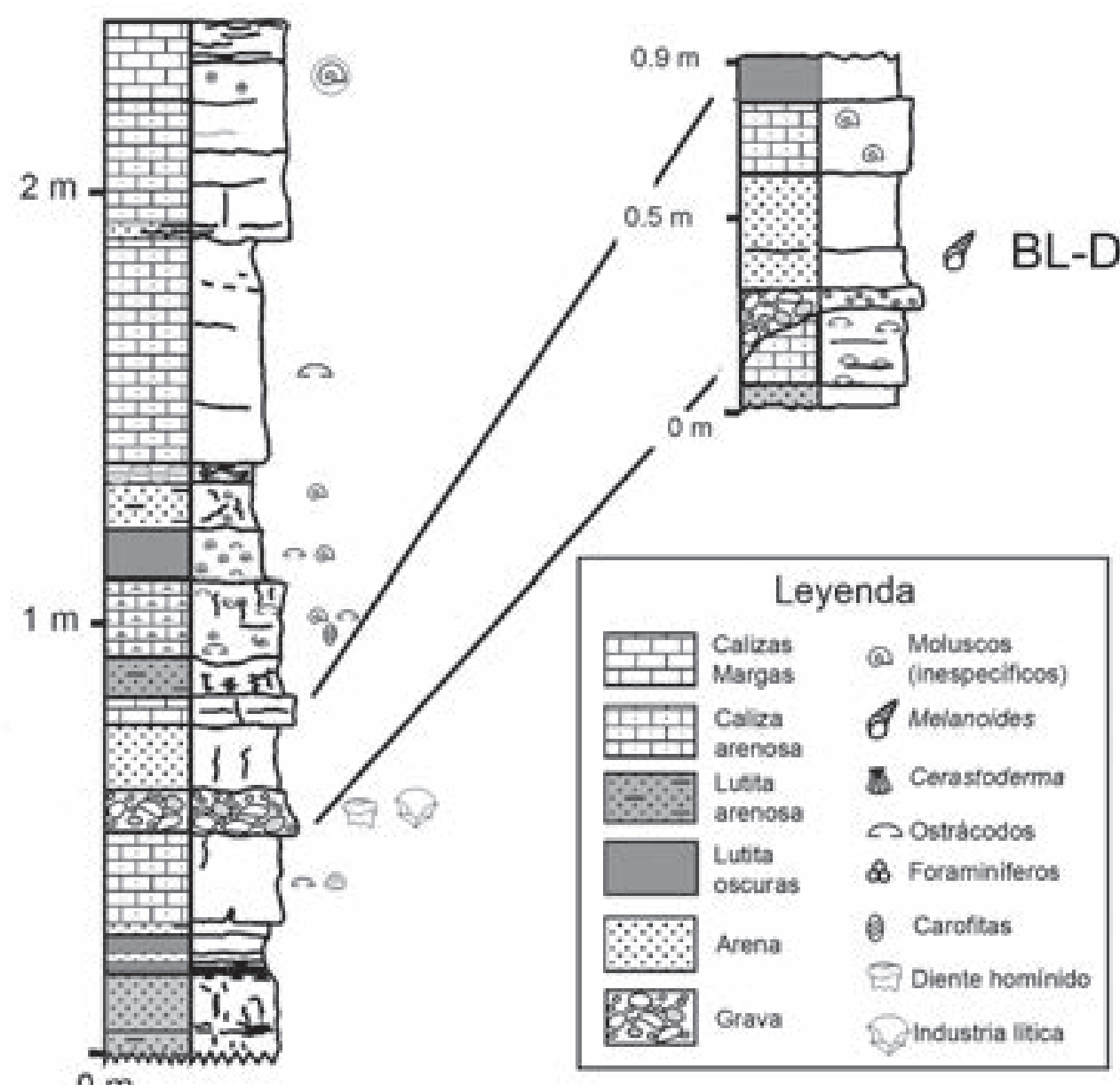

Figura 2. Columna estratigráfica de la sección que contiene el yacimiento de Barranco León. Modificado de Anadón et al. (2014).

fracciones, realizándose así la estimación sobre un total aproximado de 400 ejemplares. Con el objeto de poder analizar de un modo más objetivo el resultado se calculó la abundancia relativa de cada taxón en cada muestra. Tanto los valores resultantes del conteo como la abundancia relativa calculada en cada muestra pueden apreciarse en el apartado de discusión.

La procedencia de las muestras sobre las que se ha realizado el estudio no ha permitido disponer de suficiente información sobre aspectos tafonómicos. Tal circunstancia ha imposibilitado realizar estudios paleoecológicos detallados puesto que estos últimos precisan de estudios tafonómicos previos que informen sobre la proximidad de la asociación a una paleobicenosis (De Renzi, 1978; Acuña, 1982). Por ello, los principales resultados que se exponen se refieren al listado de los taxones localizados en cada una de las dos muestras, la discusión de su posición taxonómica, exigencias ecológicas y distribución espacio-temporal y la indicación del número concreto de individuos estudiados y los valores de abundancia relativa. Pese a la limitación que imponía la escasez de información tafonómica se ha realizado también, a partir de la composición de la asociación registrada, una interpretación paleoambiental de las condiciones existentes en el momento del depósito.

La interpretación paleoambiental se ha efectuado apoyándose en la metodología propuesta por Lozek (1964) para el Cuaternario continental. Este autor divide las especies vivientes en distintos grupos ecológicos en función del hábitat que ocupan en la actualidad, lo que permite efectuar análisis paleoecológicos de las asociaciones a partir de los requerimientos ecológicos de las especies vivas. La asignación ecológica de la especie salobre se ha realizado a partir de Pérès \& Picard (1964), quienes caracterizan las biocenosis bentónicas mediterráneas que pueden localizarse en cada zona. La atribución ecológica de las distintas especies se muestra, además de en las observaciones efectuadas sobre cada especie, en el apartado de discusión. Con todo, la interpretación paleoambiental debe ser tomada con suma cautela ante la mencionada escasez de información tafonómica. 


\section{PALEONTOLOGÍA SistemÁtica Y PALEOECOLOGÍA}

Clase GASTROPODA Cuvier, 1795

Subclase CAENOGASTROPODA Cox, 1960

Orden LITTORINIMORPHA Golikov \& Starobogatov, 1975

Superfamilia TRUNCATELLOIDEA Gray, 1840

Familia Bithyniidae Gray, 1857

Género Bithynia Leach, in Abel, 1818

Bithynia tentaculata (Linnaeus, 1758)

(Figs. 3a-3b)

Material. 25 ejemplares de la muestra BL01 L63 (MGUV-36609); 9 ejemplares de la muestra BL02 J55 (MGUV-36620).

Descripción. Concha ovalo-cónica con, en los ejemplares estudiados, hasta 3,5 vueltas preservadas de perfil poco convexo, suturas algo marcadas y crecimiento regular excepto la última, que con sección ovalada y oblicua comienza a ser más grande y ventruda. Protoconcha relativamente grande, lisa y redondeada con 1,5 vueltas. Teloconcha con ornamentación de finas líneas de crecimiento ligeramente prosoclinas. Abertura no conservada. Opérculo calcáreo, piriforme, anguloso en su parte superior y con estrías concéntricas alrededor de un núcleo central casi plano.

Observaciones. Se trata de una especie de morfología muy característica y constante a lo largo del registro fósil. Las primeras citas corresponden al Vallesiense de la Cuenca del Ródano, donde se han descrito numerosas especies de Bithynia que Truc (1971) considera como "formas" de $B$. tentaculata, taxones de rango infrasubespecífico sin validez taxonómica. Es abundante en el Turoliense austriaco (Eichkogel bei Mödling), francés (Ratavoux, Mont Lubéron) y español (Villalba Baja, El Arquillo) y ha sido citada en el propio yacimiento de Barranco León (Anadón et al., 1987, 2003), así como en otras localidades del Plioceno y Pleistoceno europeo (Royo Gómez, 1922, 1928; Wenz, 1923-1930; Ehrmann, 1933; Jaeckel, 1962; Lozek, 1964; Truc, 1971; Gittenberger et al., 1998; Albesa, 2001; Esu \& Ghinassi, 2013, etc.). En la actualidad presenta una amplia distribución geográfica extendiéndose por la mayor parte de Europa, NO de África y Siberia occidental (Fetcher \& Falkner, 1993) y ha sido introducida en Norte América (Gittenberg et al., 1998). En Andalucía ha sido localizada en varias localidades del este (Pérez-Quintero et al., 2004; Pérez-Quintero, 2007, 2011a), disponiéndose, así mismo, de registros en la misma depresión de Granada (Alonso, 1975b).

Ecología. Bithynia tentaculata es una especie calcícola común en todo tipo de masas de agua dulce con excepción de las más pequeñas. Es más abundante en los tramos tranquilos de ríos, especialmente cuando existe materia orgánica en suspensión, aunque también puede localizarse en zonas donde la corriente es más intensa. Habita sobre la vegetación, bajo las piedras cuando ésta es escasa o está ausente y sobre substrato blando, en el que se entierra en invierno (Fretter \& Graham, 1978; Gloër, 2002). Es una especie para la que se ha registrado un máximo de resistencia a la desecación de 12-14 días (Pelseneer, 1935) así como una tolerancia a la salinidad que le permite vivir en aguas de hasta el 12\%o (Adam, 1960). La especie se engloba en el grupo ecológico 10SF según Lozek (1964).

Familia Hydrobiidae Stimpson, 1856

Subfamilia Hydrobiinae Stimpson, 1865

Género Hydrobia Hartmann, 1821

Hydrobia aff. acuta (Draparnaud, 1805)

(Fig. 3g)

Material. 42 ejemplares de la muestra BL01 L63 (MGUV-36610); 7 ejemplares de la muestra BL02 J55 (MGUV-36621).

Descripción. Concha pequeña, cónica alargada, con 5,25 vueltas de crecimiento lento y regular, perfil convexo y suturas muy marcadas. Protoconcha lisa con 1,25 vueltas. Teloconcha con ornamentación a base de líneas de crecimiento prosoclinas. Última vuelta ocupando algo más de la mitad de la altura total de la concha. Ombligo en forma de fisura. Abertura ovalada equivalente casi a $1 / 3$ de la altura de la concha, ligeramente oblicua y con peristoma simple.

Observaciones. La asignación genérica de las especies de hidróbidos debe basarse, según inciden varios autores, en el estudio de la anatomía interna de las partes blandas (Radoman, 1969; Boeters, 1982; Kabat \& Hersler, 1993). El motivo se debe a que la adscripción genérica es particularmente compleja como consecuencia de la frecuencia de los fenómenos de convergencia morfológica (Radoman, 1969), la escasez de criterios morfológicos en los que basar la asignación de unas conchas concretas a un género determinado al margen de la morfología general (Kabat \& Hersler, 1993; Altaba, 1996) y la gran variabilidad intraespecífica. La taxonomía y biogeografía del género Hydrobia s.l. resulta, respectivamente, confusa y poco conocida principalmente por la dificultad que implican 

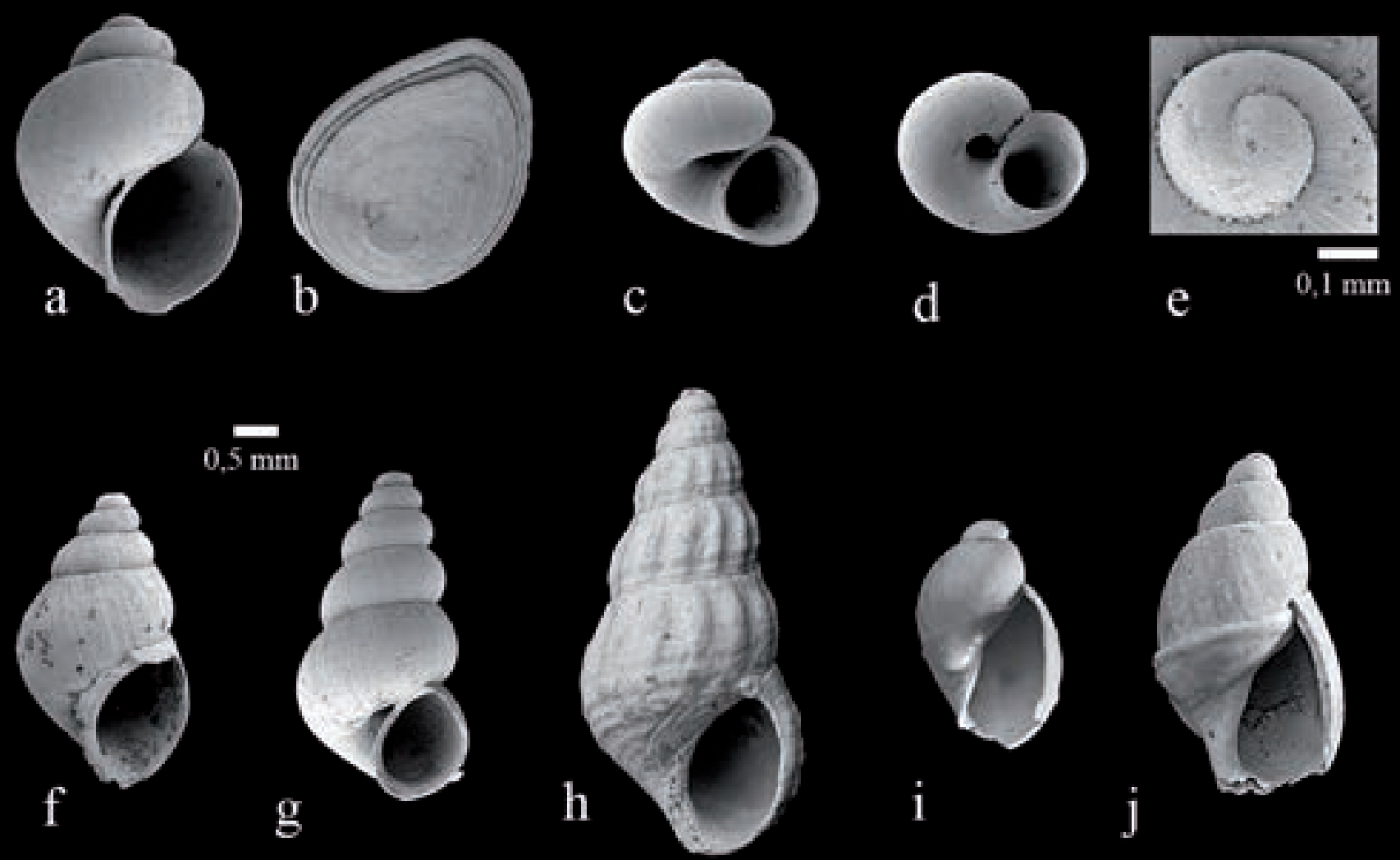

d
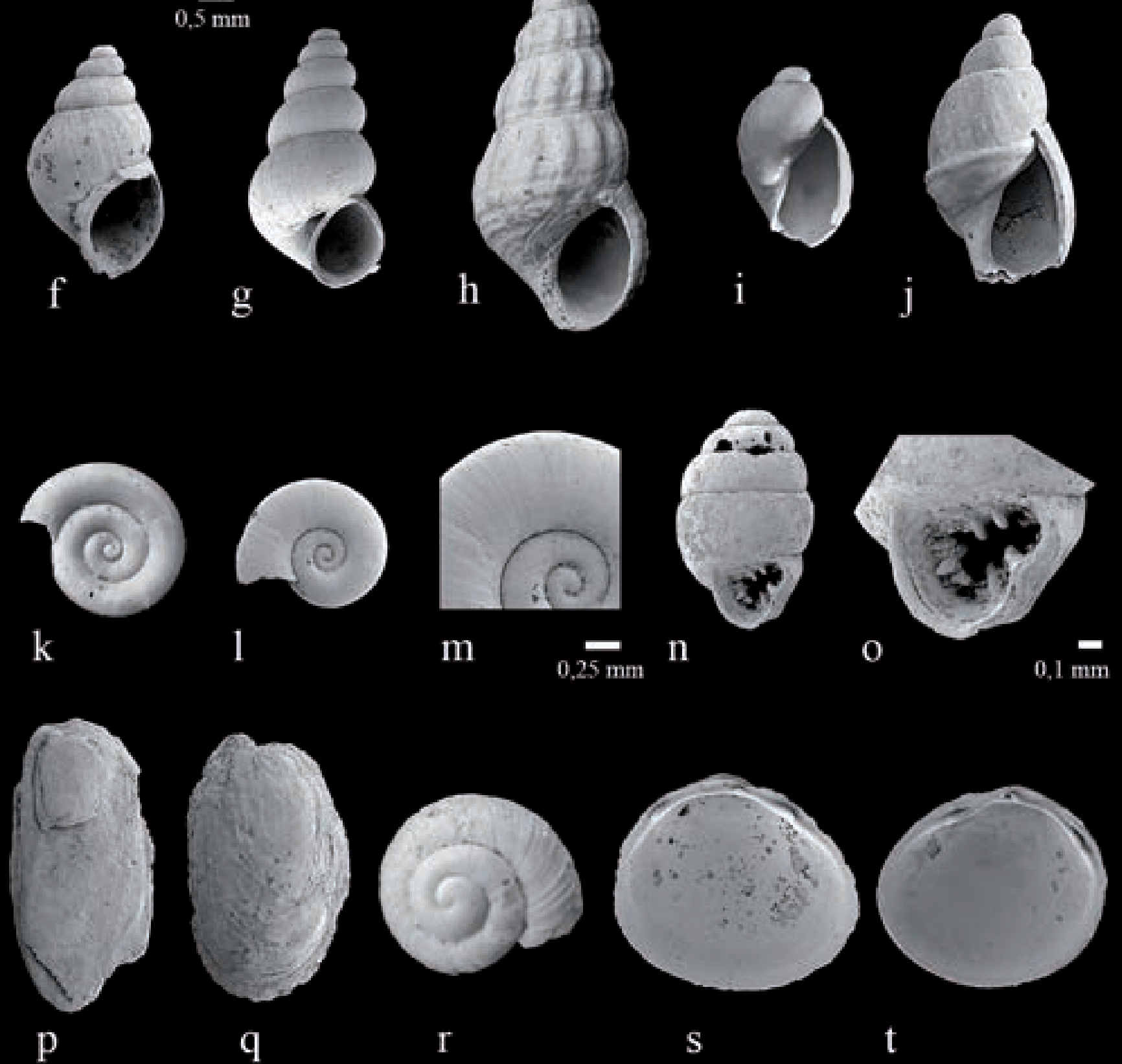

r

S

$t$ 
las radiaciones morfoestáticas de las que han surgido los taxones actuales (Wilke et al., 2000). Algunos estudios han arrojado luz tanto sobre la radiación de Hydrobia s.s. como sobre la especie concreta Hydrobia acuta y otros taxones crípticos afines, estableciendo relaciones filogenéticas y precisando las áreas de distribución (Wilke et al., 2000, 2001; Wilke \& Pfenninger, 2002). Giusti et al. (1998) designaron un neotipo de Hydrobia acuta a partir del lectotipo y paralectotipo designado por Boeters (1984a) al corresponder estos a dos especies diferentes. La morfología de los ejemplares estudiados se aproxima sólo en parte al neotipo descrito, pero coincide en buena medida con otro de los ejemplares figurados por Giusti et al. (1998) y atribuido a la especie. También se aproxima a los ejemplares figurados en Gofas et al. (2011) y procedentes de las salinas del Cabo de Gata (Almería). La especie ha sido citada por De Marfà (2007) en el yacimiento de Barranco León. Las citas existentes en la actualidad en el suroeste de Andalucía (Pérez-Quintero et al., 2004; PérezQuintero, 2011b), sureste (López et al., 2010; Gofas et al., 2011) y en la propia depresión de Granada (Alonso, 1975b) reforzarían tal asignación. Sin embargo, pequeñas divergencias morfológicas, la complejidad del grupo y la enorme variabilidad intraespecífica junto con los ambientes en los que habitualmente se localiza en la actualidad plantean dudas sobre su atribución taxonómica.

Ecología. La especie vive actualmente en lagunas costeras salobres poco profundas, en salinas y estuarios y en las zonas litorales de los mares interiores (Britton, 1985; Pantazidou et al., 2006). Se trata de un herbívoro que se alimenta de macrófitos acuáticos y habita sobre éstos y sobre sustratos arenosos y fangosos (Koutsoubas et al., 2000; Evagelopoulos et al., 2009). La especie pertenece al grupo ecológico LEE según Pérès \& Picard (1964).

Subfamilia Islamiinae Radoman, 1973

Género Islamia Radoman, 1973

Islamia sp.

(Figs. 3c-3e)
Material. 267 ejemplares de la muestra BL01 L63 (MGUV-36611); 300 ejemplares de la muestra BL02 J55 (MGUV-36622).

Descripción. Concha pequeña, valvatiforme, con 3-3,5 vueltas de espira de perfil redondeado y suturas marcadas. Protoconcha con algo más de 1,5 vueltas y ornamentación a base de fosetas. Teloconcha con ornamentación de finas líneas de crecimiento ligeramente prosoclinas. Última vuelta ocupando alrededor del $85 \%$ de la altura total de la concha. Abertura frontal y redondeada o ligeramente ovalada, equivalente a algo más del $60 \%$ de la altura de la concha, con el peristoma completo y delgado en su región externa, aunque en ocasiones ligeramente reflejado en la interna hacia un ombligo de tamaño mediano.

Observaciones. El género Islamia contiene especies difícilmente reconocibles a partir de sólo caracteres conquiológicos (Bodon \& Cianfenelli, 2012). La presencia de este género en la Península Ibérica fue confirmada por Arconada \& Ramos (2006) a partir de la revisión de las especies asignadas al género Neohoratia por Boeters (1988) tal y como apuntó Bodon et al. (2001). Resultado de esta revisión, el género Islamia amplió su número de especies y distribución geográfica reforzando la idea de que la Península Ibérica es una de las áreas más ricas en diversidad de hidróbidos de la cuenca Mediterránea (Arconada \& Ramos, 2006). Las características conquiológicas de los ejemplares estudiados entran dentro de la variabilidad de la especie Islamia henrici Arconada \& Ramos, 2006, especie actual descrita en localidades cercanas al yacimiento de Barranco León. Sin embargo, la propia variabilidad del género y particularmente de la especie junto con las reservas expuestas anteriormente sobre la determinación de los hidróbidos aconsejan prudencia por lo que la asignación que efectuamos sobre los ejemplares de Barranco León queda a nivel genérico.

Ecología. Según los autores citados las especies de Islamia habitan en fuentes no polucionadas, ricas en vegetación acuática. Los especímenes pueden localizarse sobre la vegetación, piedras, paredes húmedas y en el cieno. La especie se engloba en el grupo ecológico 10QF según Lozek (1964).

Figura 3. Moluscos localizados en las muestras estudiadas. a) Bithynia tentaculata, vista frontal. b) Bithynia tentaculata, opérculo. c) Islamia sp., vista frontal. d) Islamia sp., vista umbilical. e) Islamia sp., protoconcha. f) Pseudamnicola sp., vista frontal. g) Hydrobia aff. acuta, vista frontal. h) Melanoides tuberculata, vista frontal. i) Radix sp., vista frontal. j) Stagnicola sp., vista frontal. k) Anisus sp., vista umbilical. I) Gyraulus cf. laevis, vista umbilical. m) Gyraulus cf. laevis, detalle ornamentación. n) Vertigo antivertigo, vista frontal o) Vertigo antivertigo, detalle abertura. p) Milacidae indet. q) Milacidae indet. r) Geomitridae indet. vista apical. s) Pisidium casertanum, valva izquierda. t) Pisidium casertanum, valva derecha. La escala es la misma para todos los ejemplares, excepto en los que se indica específicamente (e, m, o). 
Subfamilia Pseudamnicolinae Radoman, 1977

Género Pseudamnicola Paulucci, 1878

\section{Pseudamnicola sp.}

(Fig. 3f)

Material. 47 ejemplares de la muestra BL01 L63 (MGUV-36612); 45 ejemplares de la muestra BL02 J55 (MGUV-36623).

Descripción. Concha pequeña, ovalado-cónica con, en los ejemplares estudiados, hasta 4,5 vueltas preservadas de perfil moderadamente convexo y suturas bastante marcadas, sección ovalada oblicua y crecimiento relativamente lento, aunque con una última vuelta más inflada. Protoconcha granulada con 1,5 vueltas. Teloconcha con ornamentación a base de líneas de crecimiento ligeramente prosoclinas. Abertura no conservada.

Observaciones. El género Pseudamnicola, con especies descritas en Europa, Asia y África, posee, en Europa, una distribución principalmente mediterránea si bien hay que apuntar también la existencia de citas en el centro y norte del continente (Delicado et al., 2012). La revisión de la familia Hydrobiidae efectuada por Boeters (1988) en la región ibero-balear situó el área de distribución del género en la mitad oriental de la Península Ibérica y en las islas Baleares. A partir tanto de la consideración de caracteres conquiológicos como de la anatomía y de la genitalia, Boeters (1988) redescribió varias especies y constató la existencia de una alta variabilidad intraespecífica dentro del género Pseudamnicola. Delicado et al. (2012) describieron nuevas especies, bastantes de ellas en el sureste de la Península Ibérica, al considerar la complementariedad de múltiples criterios tanto conquiológicos como anatómicos y moleculares. Dichos criterios complementarios, obviamente, no son aplicables a las muestras que poseemos por lo que únicamente podemos apoyarnos en los conquiológicos. Así pues, si bien, en la actualidad, en la misma población de Orce vive la especie Pseudamnicola falkneri (Boeters, 1970) y, a partir de las semejanzas conquiológicas que se aprecian, cabría la posibilidad de asignar a esta especie los ejemplares estudiados, la reducida talla de esta especie en comparación con los ejemplares estudiados y la ausencia de ejemplares completos sobre los que valorar la característica sinuosidad del peristoma desaconsejan la atribución específica. La comparación con el resto de las especies que habitan actualmente tanto en zonas próximas como en toda la región ibero-balear resulta también compleja por la mencionada variabilidad intraespecífica y ausencia de ejemplares completos, por lo que la determinación que efectuamos queda únicamente a nivel genérico a la espera de poder estudiar nuevos ejemplares en mejor estado de conservación.
Ecología. La mayoría de las especies pertenecientes al género viven actualmente en fuentes, acequias y pequeños cursos de agua permanentes, pero también en lagos y ríos (Delicado, 2013). Las especies actuales del género se engloban en el grupo ecológico 10 según Lozek (1964).

Superfamilia CERITHIOIDEA Fleming, 1822

Familia Thiaridae Gill, 1871 (1823)

Subfamila Thiarinae Gill, 1871 (1823)

Género Melanoides Olivier, 1804

Melanoides tuberculata (Müller, 1774)

(Fig. 3h)

Material. 2 ejemplares de la muestra BL01 L63 (MGUV-36613); 24 ejemplares de la muestra BL02 J55 (MGUV-36624).

Descripción. Concha turriculada de ápice puntiagudo con hasta 6 vueltas preservadas, en los ejemplares estudiados, de crecimiento lento y regular, perfil moderadamente convexo y suturas algo marcadas. Protoconcha apenas conservada. Teloconcha con ornamentación consistente en filas paralelas de tubérculos que se entrecuzan con las líneas de crecimiento. Ombligo cerrado y abertura casi ovalada y ligeramente inclinada.

Observaciones. Se trata de una especie con gran variación fenotípica en lo que se refiere a la forma, la ornamentación y la pigmentación de la concha (Pointier et al., 2003).

Es una especie termófila, nativa en las áreas tropicales y subtropicales de la región Indopacífica, sur de Asia, Arabia, Próximo Oriente y la mayor parte de África, incluyendo Madagascar, aunque falta en una amplia zona occidental, incluyendo la mayor parte de la Cuenca del Zaire (Brown, 1994). Ha sido introducida en numerosos países europeos y americanos, en los que sobrevive en ambientes naturales, cuando la temperatura del agua es suficiente, o coloniza los espacios dedicados a vegetación tropical de los jardines botánicos.

Por lo que se refiere a la Península Ibérica, en la actualidad existen múltiples localidades de distribución dispersa cuyo origen es la introducción antrópica (Martínez-Ortí, com. pers. 2017). Sin embargo, la especie está registrada en el propio yacimiento de Barranco León (Anadón et al., 1987, 2003), así como en otros yacimientos cuaternarios de Andalucía (Anadón et al., 1987; Robles, 1989) y en el Tirreniense de las Islas Baleares (Gasull, 1974) en las que, según Cuerda Barceló (1987) debió extinguirse con el descenso de la temperatura de la última glaciación. De un modo análogo, la especie ha sido localizada también en el Pleistoceno italiano (Laviano \& 
Penneta, 1980), no conociéndose registros actuales hasta la década de los 80 (Bodon et al., 1995).

Ecología. M. tuberculata es una especie eurivalente que puede colonizar biotopos muy diversos, ríos, lagos y surgencias, pero que está ausente de las masas de agua temporales. Se asocia siempre a condiciones termófilas, por lo que es considerada como un buen indicador de climas cálidos (Anadón et al., 1987; Robles, 1989; Brown, 1994). En el laboratorio se ha calculado un rango de temperaturas de supervivencia comprendido entre $18^{\circ} \mathrm{C}$ y $32^{\circ} \mathrm{C}$ (Mitchell $\&$ Brandt, 2005). Se trata de una especie eurihalina, que tolera salinidades muy elevadas de hasta el 23\%o según Plaziat \& Younis (2005) y hasta el 30\%o según Roessler et al. (1977), aunque en general aparece asociada a ambientes de baja salinidad (0,2-3\%o según Rosso (1983) in Plaziat $\&$ Younis, 2005). La especie pertenece al grupo ecológico 10 según Lozek (1964).

Subclase HETEROBRANCHIA Burmeister, 1837

Infraclase EUTHYNEURA

Subterclase TECTIPLEURA

Superorden HYGROPHILA Férussac, 1822

Superfamilia LYMNAEOIDEA Rafinesque, 1815

Familia Lymnaeidae Rafinesque, 1815

Subfamilia Amphipepleinae Pini, 1877

Género Radix Montfort, 1810

\section{Radix sp.}

(Fig. 3i)

Material. 3 ejemplares de la muestra BL01 L63 (MGUV-36614); 2 ejemplares de la muestra BL02 J55 (MGUV-36625).

Descripción. Concha conico-ovoide, con hasta 2,5 vueltas preservadas, en los ejemplares estudiados, de crecimiento rápido, perfil convexo y suturas marcadas. Protoconcha lisa con 1,25 vueltas. Teloconcha con ornamentación a base de líneas de crecimiento prosoclinas. Abertura no conservada.

Observaciones. Las especies europeas del género Radix poseen gran variación en la forma de sus conchas, circunstancia por la que algunos autores manifiestan la imposibilidad de discriminarlas si no es mediante análisis moleculares (Pfenninger et al., 2006), aunque otros han propuesto una combinación de caracteres conquiológicos y anatómicos (Schniebs et al., 2011, 2013). Las conchas estudiadas apenas muestran la protoconcha y primeras vueltas por lo que, según lo expuesto, la determinación sólo puede realizarse a nivel genérico.
Ecología. Las especies actuales de Radix habitan preferentemente aguas permanentes estancadas, dulces a mesohalinas, localizándose sobre las plantas o sobre el fondo de las riberas de los lagos y más raramente en aguas profundas o con corriente (Glöer, 2002). Este taxón se engloba en el grupo ecológico 10S(F) según Lozek (1964).

Subfamilia Lymnaeinae Rafinesque, 1815

Género Stagnicola Jeffreys, 1830

\section{Stagnicola sp.}

(Fig. 3j)

Material. 2 ejemplares de la muestra BL01 L63 (MGUV-36615); 3 ejemplares de la muestra BL02 J55 (MGUV-36626).

Descripción. Concha fusiforme y de ápice agudo con, en los ejemplares estudiados, hasta 4 vueltas preservadas de crecimiento lento, perfil moderadamente convexo y suturas bastante marcadas. Protoconcha lisa con 1,5 vueltas. Teloconcha con ornamentación de líneas de crecimiento prosoclinas. Abertura no conservada.

Observaciones. Hasta épocas recientes, la mayor parte de los autores (e.g. Kotsakis et al., 2011) sólo reconocían la existencia de una especie de este género, Stagnicola palustris (Müller, 1774), en el Cuaternario de la mayor parte de Europa, coincidiendo con el nombre que se asignaba a la especie actual. Sin embargo, estudios basados en las características anatómicas y en análisis de filogenia molecular, han mostrado que el género Stagnicola está muy diferenciado, pudiéndose reconocer hasta cinco especies europeas distintas (Bargues et al., 2001; Glöer \& MeierBrook, 1998).

Por lo que se refiere a la Península Ibérica, la mayor parte de los individuos de Stagnicola estudiados recientemente han sido asignados a S. fuscus (Pfeiffer, 1821) (Bargues et al., 2001), aunque recientemente se ha confirmado la existencia de $S$. palustris en Andalucía (Pérez-Quintero et al., 2004; Pérez-Quintero, 2009, 2011 b \& 2012).

Como sucede con los hidróbidos, no es posible diferenciar las especies de Stagnicola utilizando como único criterio la morfología de la concha, en especial cuando no se dispone de individuos adultos completos, como es nuestro caso.

Ecología. Las especies de Stagnicola prefieren las aguas permanentes, preferentemente estancadas, dulces o ligeramente oligohalinas, viviendo sobre la vegetación de macrófitos de cuyos epífitos se alimentan. (Adam, 1960; Økland, 1990; Glöer, 2002). El género Stagnicola pertenece al grupo ecológico 10P según Lozek (1964). 
Familia Planorbidae Rafinesque, 1815

Subfamilia Planorbinae Rafinesque, 1815

Género Anisus Studer, 1820

\section{Anisus sp.}

(Fig. 3k)

Material. 4 ejemplares de la muestra BL01 L63 (MGUV-36616); 4 ejemplares de la muestra BL02 J55 (MGUV-36627).

Descripción. Concha discoidal, ligeramente umbilicada por ambas caras y con hasta 3 vueltas preservadas de sección subcircular, crecimiento lento y regular, perfil ligeramente convexo y suturas bastante marcadas. Protoconcha con 1 vuelta. Teloconcha con ornamentación prosoclina en la parte umbilical y prosocirta en la apical. Abertura no conservada.

Observaciones. Los pocos ejemplares presentes en las muestras correspondían a conchas juveniles o de adultos incompletos, por lo que la determinación no ha podido realizarse más allá del nivel genérico.

Ecología. Las especies de Anisus habitan en la actualidad en aguas poco profundas, estancadas o con débil corriente, dulces u oligohalinas y con abundante vegetación. Algunas especies son muy resistentes a la desecación y habitan en masas de agua temporales, enterrándose en periodos de sequía en el cieno o entre el follaje húmedo de las helofitas (Girod et al., 1980; Glöer, 2002). La mayoría de las especies actuales del género Anisus pertenecen principalmente al grupo ecológico 10P según Lozek (1964).

Género Gyraulus Charpentier, 1837

\section{Gyraulus cf. laevis (Alder, 1838)}

(Fig. 31-3m)

Material. 2 ejemplares de la muestra BL01 L63 (MGUV-36617); 2 ejemplares de la muestra BL02 J55 (MGUV-36628).

Descripción. Concha discoidal y de contorno redondeado con hasta casi 3 vueltas preservadas, en los ejemplares estudiados, de sección algo ovalada y ligeramente oblicua, crecimiento rápido, perfil convexo y suturas marcadas. Protoconcha con algo más de 1 vuelta y ornamentación estriada. Teloconcha con ornamentación prosoclina en la parte umbilical y prosocirta en la apical. Abertura no conservada.
Observaciones. El patrón de crecimiento de los ejemplares estudiados permite atribuir al género Gyraulus a la vez que distinguirlos de los géneros Anisus o Planorbis, caracterizadas por un patrón de crecimiento más lento. La ausencia de ornamentación espiral permite además aproximarlos a G. laevis, diferenciándolos de otras especies del género, aunque la falta de individuos adultos completos impide confirmar esta determinación.

Esta especie, de distribución holártica (Glöer, 2002), ha sido localizada, entre otras provincias, en todas las del levante español comprendidas desde Cataluña hasta Murcia (Soler et al., 2006). Sin embargo, sólo ha sido colectada viva en una localidad de la depresión de Granada (Alonso, 1975b), si bien se ha recolectado también en varias de localidades de la provincia de Huelva (PérezQuintero, 2007, 2009). Robles (1989) la cita en numerosos yacimientos neógenos y cuaternarios de la Cuenca de Guadix-Baza.

Ecología. G. laevis habita en aguas someras con abundante vegetación, estancadas o con débil corriente. Frecuenta las aguas dulces, aunque tolera salinidades de entre el 3 y el 5\% (Adam, 1960; Jaeckel, 1962; Glöer \& Meier-Brook, 1998). La especie se engloba en el grupo ecológico 10S según Lozek (1964).

Superorden EUPULMONATA Haszprunar \& Huber, 1990

Orden STYLOMMATOPHORA Schmidt, 1855

Suborden HELICINA Rafinesque, 1815

Infraorden PUPILLOIDEI

Superfamilia PUPILLOIDEA Turton, 1831

Familia Vertiginidae Fitzinger, 1833

Subfamilia Vertigininae Fitzinger, 1833

Género Vertigo Müller, 1773

Vertigo antivertigo (Draparnaud, 1801)

(Fig. 3n-3o)

Material. 1 ejemplar de la muestra BL01 L63 (MGUV36618); 2 ejemplares de la muestra BL02 J55 (MGUV36629).

Descripción. Concha pequeña, ovoide ensanchada, con casi 5 vueltas muy convexas y suturas moderadamente marcadas. Protoconcha con 1,5 vueltas. Teloconcha con ornamentación a base de líneas de crecimiento prosoclinas. Última vuelta con una hendidura en el borde palatal que se corresponde con la dentición, así como con una fuerte callosidad cervical. Abertura oval con peristoma discontinuo y ligeramente reflejado y con una denticulación 
muy característica y consistente en al menos seis piezas denticulares: dos láminas parietales, dos columelares y dos palatales.

Observaciones. Se conoce en el registro fósil desde el Plioceno superior, habiendo sido citada en los yacimientos de Arenas del Rey y Fuensanta (Granada, Neógeno superior indeterminado) (Bandel et al., 2000), el yacimiento de El Arquillo 3 (Teruel, zona MN15) (Albesa, 2001), así como en el de Villarroya (La Rioja, zona MN16) (Rodríguez-Lázaro et al., 1997), en diversas localidades plio-pleistocenas de la Cuenca de Cúllar-Baza (Robles, 1989), en el Plio-Pleistoceno de la cuenca italiana de Mugello (Benvenuti et al., 1998) y en el yacimiento de Montagny-les-Beaune (Francia, zona MN17) (Schlickum \& Puisségur, 1978). En la actualidad posee distribución paleártica (Kerney \& Cameron, 1999), habiendo sido recogida enterrada en choperas en la depresión de Granada (Alonso, 1975b).

Ecología. Se trata de una especie terrestre hidrófila que habita en los marjales, frecuentemente bajo las hojas muertas de las plantas palustres y en el mantillo del borde de lagos y charcas (Kerney et al., 1983). La especie se engloba en el grupo ecológico 9P según Lozek (1964).

\section{Infraorden LIMACOIDEI}

Superfamilia PARMACELLOIDEA Fischer, 1856 (1855)

Familia Milacidae Ellis, 1926

Milacidae indeterminados

(Figs. 3p-3q)

Material. 6 ejemplares de la muestra BL02 J55 (MGUV-36630).

Descripción. Limacela en forma de placa simétrica entre elíptica y subrectangular. Delgada, de perfil bajo que aumenta desde el margen anterior hacia una protoconcha bastante ancha y casi centrada. Superficie dorsal ornamentada con líneas de crecimiento concéntricas. Superficie ventral lisa y ligeramente deprimida.

Observaciones. Las babosas están representadas en Barranco León por sus limacelas, placas calcáreas internas resultantes de la reducción de la concha en el proceso de limacización. La posición centrada del núcleo permite diferenciar los integrantes de las familias Milacidae y Boetgerillidae de los de las familias Limacidae y Agriolimacidae. Dentro de las primeras, las limacelas de Milacidae son proporcionalmente mucho más gruesas y están mejor desarrolladas que las de Boetgerillidae (Evans,
1972; Borredà, 1996), lo que justifica nuestra adscripción. La determinación genérica rara vez es posible.

Limacelas de esta familia han sido citadas por Robles (1989) en numerosos yacimientos del Plio-Pleistoceno de la Cuenca de Cúllar-Baza, así como de Arionidae por parte de De Marfà (2007) en el propio yacimiento de Barranco León.

Ecología. La mayor parte de las especies tienen, por la ausencia de concha externa, fuerte dependencia de la humedad, por lo que es frecuente recogerlas en zonas húmedas $\mathrm{y}$ en los bordes de las masas de agua (Borredà, 1996). Las especies de la familia Milacidae se engloban en el grupo ecológico 7M según Lozek (1964).

\section{Infraorden HELICOIDEI}

Superfamilia HELICOIDEA Rafinesque, 1815

Familia Geomitridae Boettger, 1909

Geomitridae indeterminado

(Fig. 3r)

Material. 1 ejemplar de la muestra BL01 L63 (MGUV36619); 2 ejemplares de la muestra BL02 J55 (MGUV36631).

Descripción. Concha conico-deprimida con hasta 3,5 vueltas preservadas de perfil redondeado, aunque algo aquillado en alguno de los ejemplares, crecimiento regular y lento y suturas moderadamente marcadas. Protoconcha con 1,5 vueltas y un claro límite con la teloconcha. Teloconcha con ornamentación a base de robustas costulaciones irregulares en la parte apical y más tenues en la umbilical. Ombligo mediano y profundo. Abertura no conservada.

Observaciones. Sólo hemos dispuesto de escasos ejemplares incompletos que conservaban únicamente la protoconcha y el inicio de la teloconcha, lo que ha impedido determinarlos incluso a nivel genérico. El grosor de la concha y la ornamentación descrita aproximan nuestro material a especies de hábitos xerófilos pertenecientes a la familia Geomitridae como Cernuella, Helicella, Candidula, Trochoidea y Xerocrassa.

Anadón et al. (1987) cita la presencia de Helicella sp. en uno de los perfiles estudiados en el área de Barranco León mientras que Robles (1989) lo hace en varios yacimientos plio-pleistocenos de la Cuenca de CúllarBaza. De Marfà (2007) cita Trochoidea indet. en el propio yacimiento de Barranco León objeto de este estudio.

Ecología. Estas especies, con un carácter xerófilo muy marcado (Kerney et al., 1983), constituyen hoy 
en día el conjunto más representativo y más numeroso de la malacofauna circummediterránea, colonizando los espacios abiertos o con vegetación degradada (Dubar \& Magnin, 1992). Especies de los géneros indicados han sido colectadas habitualmente tanto en el matorral mediterráneo como en bosques de pinaceas $\mathrm{y}$, en menor medida, qüercíneas (Martínez-Ortí, 1999). Las especies actuales de estos géneros se engloban en el grupo ecológico 4S según Lozek (1964).

\section{Clase BIVALVIA Linnaeus 1758}

Subclase HETERODONTA Neumayr, 1883

Infraclase EUHETERODONTA

Superorden IMPARIDENTIA Bieler et al., 2014

Orden SPHAERIIDA Lemer et al., 2019

Superfamilia SPHAERIOIDEA Deshayes, 1855 (1820)

Familia Sphaeriidae Deshayes, 1855 (1820)

Subfamilia Sphaeriinae Deshayes, 1855 (1820)

Género Pisidium Pfeiffer, 1821

Pisidium casertanum (Poli, 1791)

(Figs. 3s-3t)

Material. 10 ejemplares de la muestra BL02 J55 (MGUV-36632).

Descripción. Concha pequeña con silueta entre ovalada y triangular, siendo la región anterior algo más larga que la posterior. Umbos anchos pero poco prominentes. Estriación fina e irregular. Charnela generalmente ancha y variable. Valva izquierda con un diente cardinal inferior arqueado y otro superior habitualmente más posterior, oblicuo y ligeramente curvo. Valva derecha con un diente cardinal curvo con el extremo posterior acampanado. Dientes laterales de ambas valvas igualmente variables aunque, por lo general, bien desarrollados.

Observaciones. Se trata del único bivalvo presente en las muestras estudiadas. P. casertanum es una especie con morfología muy variable, si bien los caracteres observados en nuestros ejemplares e indicados en la descripción, concuerdan con los de esta especie.

Ecología. Se trata de una especie muy eurivalente que coloniza todos los tipos de medios acuáticos, incluso los más extremos: pequeños cursos de agua temporales, masas de agua efímeras, lagos alpinos aislados, etc. Soporta importantes fluctuaciones de la temperatura del agua y salinidades hasta del 3\%o (Jaeckel, 1962; Mouthon \& Kuiper, 1987). Se trata de una especie cosmopolita de amplia distribución en la Península Ibérica (Soler et al., 2006), siendo el bivalvo viviente más abundante en la depresión de Granada (Alonso, 1975a). La especie pertenece al grupo ecológico 10FPpQ según Lozek (1964).

\section{DISCUSIÓN}

Los trabajos previos que también aportaron un listado de moluscos para la capa D del yacimiento de Barranco León, Anadón et al. (2003) y De Marfà (2007), citaron respectivamente cuatro y siete taxones. Puesto que sólo una especie coincidía entre ellos, diez era el número de especies conocidas hasta la actualidad para la mencionada capa. El presente trabajo, con hasta 13 especies diferentes localizadas, de las que tres son coincidentes con las de los trabajos previos y diez son citas nuevas, supone un importante incremento en el número de especies citadas en la capa $\mathrm{D}$ del yacimiento de Barranco León, alcanzándose con ello la cifra de 20 taxones diferentes.

Por lo que respecta a las especies localizadas en este estudio, la muestra BL01 L63 contiene un total de 11 especies diferentes, de las cuales cinco han podido ser determinadas a nivel específico (Bithynia tentaculata, Hydrobia aff. acuta, Melanoides tuberculata, Gyraulus cf. laevis y Vertigo antivertigo), otras cinco a nivel genérico (Islamia sp., Pseudamnicola sp., Radix sp., Stagnicola sp. y Anisus sp.) y una sólo a nivel de familia (Geomitridae indet.). La muestra BL02 J55 contiene 13 especies, de las que 11 coinciden con las de la muestra BL01 y otras dos (Milacidae indet. y Pisidium casertanum) son específicas de esta muestra. El listado de taxones localizados en cada muestra, junto con los citados para la propia capa D, así como también en el área de Barranco León en los trabajos previos se expone en la Tabla 1.

La asociación de moluscos registrada en las muestras estudiadas, cuyos valores del conteo y abundancia relativa calculada en cada una se muestran en la Tabla 2, se caracteriza por la predominancia de especies acuáticas junto a escasos representantes de taxones terrestres, lo que le confiere un marcado carácter acuático.

Por lo que se refiere a los moluscos acuáticos, destacan, con valor de abundancia relativa del 94,6\%, las especies de prosobranquios que mayoritariamente pertenecen a géneros caracterizados por su elevada tolerancia a la salinidad y, en términos generales, escasa resistencia a la desecación.

Dentro de los prosobranquios, es especialmente significativa la presencia de tres especies de hidróbidos, lo que apoya la idea de que la Península Ibérica, y en especial Andalucía Oriental, es una de las áreas con mayor diversidad y endemicidad de hidróbidos (Arconada \& Ramos, 2003). Relevante es en concreto la presencia de especies de los géneros Islamia y Pseudamnicola, taxones de los que se han descrito numerosas especies de carácter endémico en el sureste de la Península Ibérica 
Tabla 1. Taxones citados en el área del barranco León por los trabajos indicados.

\begin{tabular}{|c|c|c|c|c|}
\hline Taxones & Anadón et al. (1987) & Anadón et al. (2003) & De Marfià (2007) & Este trabajo \\
\hline Viviparus sp. & $\mathrm{X}$ & & & \\
\hline Viviparidae indet. & $\mathrm{X}$ & & & \\
\hline Bithynia tentaculata & cf. & cf. & & $\mathrm{X}$ \\
\hline Bithyniidae indet. & $\mathrm{x}$ & & & \\
\hline Hydrobia acuta & & & $\mathrm{x}$ & aff. \\
\hline Hydrobiidae indet. & $\mathrm{x}$ & & & \\
\hline Islamia minuta & & & $\mathrm{x}$ & \\
\hline Islamia sp. & & & & $\mathrm{X}$ \\
\hline Mercuria emiliana & & & $\mathrm{x}$ & \\
\hline Pseudamnicola sp. & & & & $\mathrm{X}$ \\
\hline Melanoides tuberculata & aff. & aff. & aff. & $\mathrm{x}$ \\
\hline Melanoides sp. & $\mathrm{x}$ & & & \\
\hline Valvata (Cincinna) cf. nilotica & $\mathrm{x}$ & & & \\
\hline Valvata sp. & $\mathrm{x}$ & $\mathrm{x}$ & & \\
\hline Bulinus (Physopsis) cf. truncatus & $\mathrm{x}$ & & & \\
\hline Lymnaea (Radix) peregra, & $\mathrm{x}$ & & & \\
\hline Lymnaea (Stagnicola) palustris, & $\mathrm{x}$ & & & \\
\hline Radix sp. & & & & $\mathrm{X}$ \\
\hline Stagnicola sp. & & & & $\mathrm{X}$ \\
\hline Ancylus cf. lacustris, & $\mathrm{x}$ & & & \\
\hline Ferrissia sp. & $\mathrm{x}$ & & & \\
\hline Anisus sp. & & & & $\mathrm{X}$ \\
\hline Gyraulus cf. albus & $\mathrm{X}$ & & & \\
\hline Gyraulus cf. laevis & & & & $\mathrm{X}$ \\
\hline Gyraulus sp. & $\mathrm{X}$ & & & \\
\hline Armiger cf. crista & $\mathrm{x}$ & & & \\
\hline Planorbidae indet. & & $\mathrm{x}$ & & \\
\hline Vertigo antivertigo & & & & $\mathrm{X}$ \\
\hline Milacidae indet. & & & & $\mathrm{X}$ \\
\hline Arionidae indet. & & & $\mathrm{x}$ & \\
\hline Helicella sp. & $\mathrm{x}$ & & & \\
\hline Trochoidea indet. & & & $\mathrm{x}$ & \\
\hline Geomitridae indet. & & & & $\mathrm{X}$ \\
\hline Pisidium casertanum & & & & $\mathrm{X}$ \\
\hline Pisidium sp. & $\mathrm{X}$ & & $\mathrm{x}$ & \\
\hline
\end{tabular}

(Boeters, 1970, 1984b, 1988, 1999; Arconada \& Ramos, 2006; Delicado et al., 2012; Boeters et al., 2015). Algunos autores indican que estas especies debieron de surgir como consecuencia de fenómenos tectónicos que, iniciados en el Neógeno, persistieron durante el Cuaternario y originaron cambios paleobiogeográficos y la aparición de nuevos espacios ecológicos que posibilitaron fenómenos de especiación (Delicado et al., 2012). Evidencias de la existencia de fenómenos tectónicos, y particularmente de su persistencia durante el cuaternario, son la densa y compleja red de fallas existente, así como las altas tasas de hundimiento en determinados periodos y la presencia de paleosismitas en el registro sedimentario (García-Aguilar et al., 2014). La detección de especies endémicas tanto del género Islamia como Pseudamnicola en el Pleistoceno inferior de la región es coherente con esta posibilidad, teniendo su origen en los nuevos espacios ecológicos que surgieron como consecuencia de la actividad tectónica. 
Tabla 2. Taxones localizados en cada una de las muestras estudiadas con indicación del número de ejemplares estudiados, abundancia relativa en la muestra y grupo ecológico al que pertenecen. 4S: especies de áreas secas y soleadas desprovistas de vegetación arbórea; 7M: especies mesófilas que pueden vivir en lugares húmedos o secos; 9P: especies fuertemente higrófilas que viven en marismas o en lugares similares muy húmedos, generalmente muy cerca del agua; 10: especies acuáticas que pueden vivir en diferentes biotopos o cuyo hábitat no puede determinarse; 10S: especies acuáticas de aguas tranquilas, pequeñas marismas, estanques grandes o lagos; 10SF: especies acuáticas de agua inmóvil o en movimiento; 10S(F): especies acuáticas de agua inmóvil aunque, en menor medida, también en movimiento;10P: especies acuáticas de humedales con aguas poco profundas y ricas vegetación; 10QF: especies acuáticas de manantiales o de aguas en movimiento; 10FPpQ: especies acuáticas que viven tanto en aguas en movimiento como en marismas periódicas o fuentes; LEE: especies lagunares de biocenosis euritermas y eurihalinas.

\begin{tabular}{|c|c|c|c|c|c|}
\hline \multirow[t]{2}{*}{ Especies } & \multicolumn{2}{|c|}{ BL01 } & \multicolumn{2}{|c|}{ BL02 } & \multirow[b]{2}{*}{ Grupo ecológice } \\
\hline & $\mathrm{N}^{\mathrm{o}}$ indiv. & $\operatorname{Ar}(\%)$ & $\mathrm{N}^{\mathrm{o}}$ indiv. & $\operatorname{Ar}(\%)$ & \\
\hline Bithynia tentaculata & 25 & 6,31 & 9 & 2,16 & $10 \mathrm{SF}$ \\
\hline Hydrobia aff. acuta & 42 & 10,6 & 7 & 1,68 & LEE \\
\hline Islamia sp. & 267 & 67,4 & 300 & 72,1 & $10 \mathrm{QF}$ \\
\hline Pseudamnicola sp. & 47 & 11,9 & 45 & 10,8 & 10 \\
\hline Melanoides tuberculata & 2 & 0,51 & 24 & 5,77 & 10 \\
\hline Radix sp. & 3 & 0,76 & 2 & 0,48 & $10 \mathrm{~S}(\mathrm{~F})$ \\
\hline Stagnicola sp. & 2 & 0,51 & 3 & 0,72 & $10 \mathrm{P}$ \\
\hline Anisus sp. & 4 & 1,01 & 4 & 0,96 & $10 \mathrm{P}$ \\
\hline Gyraulus cf. laevis & 2 & 0,51 & 2 & 0,48 & $10 \mathrm{~S}$ \\
\hline Vertigo antivertigo & 1 & 0,25 & 2 & 0,48 & $9 \mathrm{P}$ \\
\hline Milacidae indet. & & 0 & 6 & 1,44 & $7 \mathrm{M}$ \\
\hline Geomitridae indet. & 1 & 0,25 & 2 & 0,48 & $4 \mathrm{~S}$ \\
\hline Pisidium casertanum & & 0 & 10 & 2,4 & $10 \mathrm{FPpQ}$ \\
\hline Total & 396 & 100 & 416 & 100 & \\
\hline
\end{tabular}

Nuevos muestreos en los otros yacimientos cuaternarios de la cuenca podrían arrojar luz sobre la filogenia de estas especies.

Aunque con un valor de abundancia relativa del 2,7\% (muy inferior al de los prosobranquios), la asociación estudiada también se encuentra integrada por especies de basomatóforos que se caracterizan por soportar salinidades de hasta un 5\%, así como la desecación, aunque prefieren aguas dulces, permanentes y preferentemente estancadas. Interesante resulta la presencia en las muestras de Gyraulus cf. laevis, especie citada en la Cuenca de Guadix-Baza en diversos yacimientos neógenos y cuaternarios (Robles, 1989) pero de escasa localización en la actualidad (Alonso, $1975 \mathrm{~b}$ ) pese a que si ha sido localizada en regiones próximas (Pérez-Quintero, 2007). Con todo, se trata de una especie cuyo registro puede ser considerado como continuo desde el Neógeno hasta la actualidad.

El análisis de la combinación de prosobranquios y basomatóforos presentes en las muestras estudiadas aporta información adicional. La diversidad y elevada abundancia de los primeros evidencia una masa de agua permanente que algunos autores (Oms et al., 2011) indican persistía gracias a la combinación de aportes meteóricos subterráneos y superficiales. La presencia de los segundos sugiere un régimen léntico y una escasa profundidad al menos en los márgenes del lago, así como la existencia de macrófitos sobre los cuales vivirían adheridos. La consideración de ambos en su conjunto sugeriría así mismo aguas como máximo oligohalinas, coincidiendo con lo expuesto por Anadón et al. (2014).

La presencia de Melanoides tuberculata proporcionaría a las muestras un marcado carácter termófilo, aunque las observaciones sobre el estado de conservación sugieren una posible historia tafonómica diferente en comparación con el resto de taxones presentes en las muestras. Esta circunstancia también ha sido observada por Anadón et al. (2014) al detectar en las conchas de esta especie isótopos asociados al crecimiento en aguas de manantiales termales y su ausencia en las conchas de otras especies que acompañaban a la primera en las muestras que estudiaron. Todo ello evidenciaría un transporte de las conchas de $M$. tuberculata, desde ambientes termales próximos, lo que obligaría a tenerlo en consideración al realizar las interpretaciones paleoambientales. La presencia de esta especie termófila resultaría coherente con la existencia de aportes termales, como indica se darían García-Aguilar et al. 
(2014), habitando sólo en aquellos puntos del humedal en el que se encontrasen unas surgencias en las que, según Anadón et al. (2014), la temperatura del agua estaría alrededor de los $25^{\circ} \mathrm{C}$ frente al máximo de $18^{\circ} \mathrm{C}$ calculado para el resto de las aguas.

En lo que respecta a los taxones terrestres, cuya presencia puede explicarse por arrastres debidos a fenómenos de arroyada, su abundancia relativa es, con un valor del 1,5\%, escasa. Esta circunstancia puede estar relacionada con los reducidos aportes que se darían ante los relativamente bajos fenómenos de escorrentía que algunos autores sugieren ocurrirían (García-Aguilar et al., 2014) a partir de la ausencia de facies detríticas en la unidad (Arribas \& Palmqvist, 1998 in García-Aguilar et al., 2014). La localización de estos taxones aporta valiosa información sobre los paleoambientes circundantes al lago. La existencia de ejemplares de Vertigo antivertigo y de Milacidae indet. evidenciaría, por el carácter higrófilo de ambos taxones, que suelen habitar frecuentemente bajo la hojarasca de los bordes de las masas de agua, la existencia de una franja de vegetación litoral. Por otra parte, la presencia de ejemplares de Geomitridae indet. apuntan, en consonancia con lo expuesto por otros autores (Agustí et al., 2010), la existencia de espacios abiertos más allá de la franja de vegetación litoral en los que las condiciones ambientales tendrían un carácter más xerófilo.

\section{CONCLUSIONES}

La asociación de moluscos registrada en las dos muestras estudiadas en la capa D del yacimiento de Barranco León se encuentra integrada por especies mayoritariamente acuáticas, aunque también por algunas terrestres.

Los taxones predominantes corresponden, principalmente, a especies de prosobranquios acuáticos caracterizados por la elevada tolerancia a la salinidad y escasa resistencia a la desecación. Destacable es el hallazgo de Islamia sp. y Pseudamnicola sp. por su probable carácter endémico y la presencia de Melanoides tuberculata por su carácter termófilo. Relevante es también la existencia de basomatóforos, entre los que destaca Gyraulus cf. laevis, por la información que suministran acerca de la presencia de macrófitos. Los taxones terrestres corresponden tanto a especies higrófilas, como Vertigo antivertigo y Milacidae indet., ligadas a determinados ambientes más próximos a las masas de agua, como a otras xerófilas pertenecientes a la familia Geomitridae y más propias de espacios abiertos propios del matorral mediterráneo.

La presencia de todas las especies estudiadas resulta coherente con la existencia de un lago permanente, oligohalino, de régimen léntico, de escasa profundidad, con presencia de macrófitos y existencia de surgencias termales en las que habitarían las especies de carácter termófilo. Este lago estaría rodeado por vegetación litoral en lo que constituiría una franja de carácter higrófilo y se encontraría enmarcado en un espacio abierto en el que las condiciones climáticas serían de carácter más xérico y en el que encontraríamos un matorral mediterráneo salpicado de agrupaciones de árboles.

\section{AGRADECIMIENTOS}

Los autores quieren agradecer tanto a Andrés Santos como al Dr. Jordi Agustí el haber brindado la posibilidad de estudiar el material. Particularmente, el primer autor quiere aprovechar estas líneas para expresar al segundo autor de este trabajo, recientemente fallecido, todo su agradecimiento y consideración.

\section{REFERENCIAS}

Abel, C. 1818. Narrative of a Journey in the Interior of China, and of a Voyage to and from that Country, in the Years 1816 and 1817; Containing an Account of the most Interesting Transactions of Lord Amherst's Embassy to the Court of Pekin, and Observations on the Countries which it Visited. Illustrated by Maps and other Engravings. Longman, Hurst, Rees, Orme and Brown, London, xvi +420 pp.

Acuña, J.D. 1982. Algunas regularidades taxonómicas y paleoecológicas en una secuencia tortonienses de Torreblanca (Castellón). Estudios geológicos, 38, 61-73.

Adam, W. 1960. Faune de Belgique. Mollusques. Tome I. Mollusques Terrestres et Dulcicoles. Patrimoine de l'Institut royal des Sciences naturelles de Belgique, Bruxelles, 402 pp.

Agustí, J., Blain, H.-A.A., Furió, M., De Marfá, R. \& Santos-Cubedo, A. 2010. The early Pleistocene small vertebrate succession from the Orce region (GuadixBaza Basin, SE Spain) and its bearing on the first human occupation of Europe. Quaternary International, 223, 162-169; doi: 10.1016/j.quaint.2009.12.011.

Albesa, J. 2001. Moluscos neógenos de la Depresión de Teruel. Tesis Doctoral, Universitat de València, 300 pp. (inédita).

Alder, J. 1838. Supplement to a catalogue of the land and fresh-water testaceous Mollusca, found in the vicinity of Newcastle. Transactions of the Natural History Society of Northumberland and Durham, 1, 337-342.

Alonso, M.R. 1975a. El género Pisidium Pfeiffer (Mollusca, Sphaeriidae) en la depresión de Granada, España. Cuadernos de Ciencias Biológicas, 4, 97-104.

Alonso, M.R. 1975b. Moluscos terrestres y dulceacuícolas de la depresión de Granada (España) y sus alrededores. Cuadernos de Ciencias Biológicas, 4, 125-157. 
Altaba, C.R. 1996. Counting species names. Nature, 380, 488-489.

Anadón, P., Juliá, R., de Deckker, P., Rosso, J.C. \& SouliéMärsche, I. 1987. Contribución a la Paleolimnología del Pleistoceno inferior de la Cuenca de Baza (sector Orce-Venta Micena). Paleontologia i Evolució, Memoria Especial, 1, 35-72.

Anadón, P., Juliá, R. \& Oms, O. 2003. Estratigrafía y estudio sedimentológico preliminar de diversos afloramientos en Barranco León y Fuente Nueva (Orce, Granada). In: El Pleistoceno Inferior de Barranco León y Fuente Nueva 3, Orce (Granada) (coords. Toro, I., Agustí, J. \& MartínezNavarro, B.). Memoria científica campañas 1999-2002. Arqueología Monografías, 17, 47-72. Junta de Andalucía, Sevilla.

Anadón, P., Oms, O., Riera, V. \& Julià, R. 2014. The geochemistry of biogenic carbonates as a paleoenvironmental tool for the Lower Pleistocene Barranco León sequence (BL-5D, Baza Basin, Spain). Quaternary International, 389, 70-83; doi: 10.1016/j.quaint.2014.09.062.

Arconada, B. \& Ramos, M.A. 2001. New data on Hydrobiidae systematics: two new genera from the Iberian Peninsula. Journal of Natural History, 35, 949-984; doi: 10.1080/002229301300323884.

Arconada, B. \& Ramos, M.A. 2003. The Ibero-Balearic region: one of the areas of highest Hydrobiidae (Gastropoda, Prosobranchia, Rissooidea) diversity in Europe. Graellsia, 59, 91-104.

Arconada, B. \& Ramos, M.A. 2006. Revision of the genus Islamia Radoman, 1973 (Gastropoda, Caenogastropoda, Hydrobiidae), on the Iberian Peninsula and description of two new genera and three new species. Malacologia, 48, 77-132.

Arconada, B., Delicado, D. \& Ramos, M.A. 2007a. A new genus and two new species of Hydrobiidae (Mollusca, Caenogastropoda) from the Iberian Peninsula. Journal of Natural History, 41, 2007-2035; doi: 10.1080/00222930701529273.

Arconada, B., Rolán, E. \& Boeters, H.D. 2007b. A revision of the genus Alzoniella Giusti \& Bodon, 1984 (Gastropoda, Caenogastropoda, Hydrobiidae) on the Iberian Peninsula and its implications for the systematics of the European hydrobiid fauna. Basteria, 71, 113-156.

Arribas, A. \& Palmqvist, P. 1998. Taphonomy and paleoecology of an assemblage of large mammals: hyaenid activity in the lower Pleistocene site at Venta Micena (Orce, Guadix-Baza basin, Granada, Spain). Geobios, 31, 3-47; doi: 10.1016/ S0016-6995(98)80056-9.

Arribas, A. \& Palmqvist, P. 2002. The first human dispersal to Europe: remarks on the archaeological and palaeoanthropological record from Orce (Guadix-Baza basin, southeastern Spain). Human Evolution, 17, 55-78; doi: 10.1007/BF02436429.

Bandel, K., Reicherter, K., Dyrssen, U. \& Reiss, U. 2000. Late Neogene lacustrine sedimentary facies and gastropod assemblages (Granada Basin, southern Spain). Mitteilungen aus dem Geologisch-Paläontologischen Institut, 84, 111-130.
Bargues, M.D., Vigo, M., Horak, P., Dvorak, J., Pointier, J.P., Jackievicz, M., Meier-Brook, C. \& Mas-Coma, S. 2001. European Lymnaeidae (Mollusca, Gastropoda), intermediate hosts of tremadiases, based on nuclear ribosomal DNA ITS2 sequences. Infection, Genetics and Evolution, 1, 85-107.

Benvenuti, M., Esu, D., Geraci, V. \& Ghetti, P. 1998. The molluscan assemblages in the fluvio-lacustrine succession of the Plio-Pleistocene Mugello Basin (Tuscany, Italy). Rivista Italiana di Paleontologia e Stratigrafia, 104, 263278; doi: 10.13130/2039-4942/5334.

Bieler, R., Mikkelsen, P.M., Collins, T.M., Glover, E.A., González, V.L., Graf, D.L., Harper, E.M., Healy, J., Kawauchi, G.Y., Sharma, P.P., Staubach, S., Strong, E.E., Taylor, J.D., Tëmkin, I., Zardus, J.D., Clark, S., Guzmán, A., McIntyre, E., Sharp, P. \& Giribet, G. 2014. Investigating the Bivalve Tree of Life an exemplar-based approach combining molecular and novel morphological characters. Invertebrate Systematics, 28, 32-115.

Bodon, M. \& Cianfanelli, S. 2012. Il genere Islamia Radoman, 1973, nell'Italia centro-settentrionale (Gastropoda: Hydrobiidae). Bolletino Malacologico, 48, 1-37.

Bodon, M., Manganelli, L., Favilli, F. \& Giusti, F. 1995. Prosobranchia Archaeogastropoda Neritimorpha (generi 013-014); Prosobranchia Caenogastropoda Architaenioglossa (generi 060-065); Prosobranchia Caenogastropoda Neotaenioglossa p.p. (generi 070- 071, 077, 095-126); Heterobranchia Heterostropha p.p. (genere 294). In: Checklist delle Specie della Fauna d'Italia, 14 (Gastropoda Prosobranchia, Heterobranchia) (eds Minelli A., Ruffo, S. \& La Posta, S.). Calderini, Bologna, 1-60.

Bodon, M., Manganelli, G. \& Giusti, F. 2001. A survey of the European valvatiform hydrobiid genera, with special reference to Hauffenia Pollonera, 1898 (Gastropoda: Hydrobiidae). Malacologia, 43, 103-215.

Boeters, H.D. 1970. Corrosella n. gen. [Westeuropäische Hydrobiidae, 3] (Prosobranchia, Hydrobiidae). Journal de Conchyliologie, 108, 63-69.

Boeters, H.D. 1982. Species concept of Prosobranchia freshwater molluscs in Western Europa. 2. Malacologia, 22, 489-501.

Boeters, H.D. 1984a. Zur Identitat des Hydrobia-Typus (Prosobranchia: Hydrobiidae). Heldia, 1, 3-5.

Boeters, H.D. 1984b. Unbekannte westeuropäische Prosobranchia, 6. Heldia, 1, 9-10.

Boeters, H.D. 1988. Moitessieriidae und Hydrobiidae in Spanien und Portugal. Archiv für Molluskenkunde, 118, 181-261.

Boeters, H.D. 1999. Alzoniella navarrensis n. sp., Pseudamnicola (Corrosella) hydrobiopsis n. sp. and the type species of Pseudamnicola Paulucci, 1877. Unknown West European Prosobranchia, 9. Basteria, 63, 77-81.

Boeters, H.D. 2003. Supplementary notes on Moitessieriidae and Hydrobiidae from the Iberian Peninsula (Gastropoda, Caenogastropoda). Basteria, 67, 1-41.

Boeters, H.D., Callot-Girardi, H. \& Knebelsberer, T. 2015. News of Pseudamnicola (Corrosella) of Spain and France (Mollusca: Gastropoda: Truncatelloidea). Folia Malacologica, 23, 95-119; doi: 10.12657/folmal.023.007. 
Boettger, C.R. 1909. Ein Beitrag zur Erforschung der europäischen Heliciden. Nachrichtsblatt der Deutschen Malakozoologischen Gesellschaft, 41, 1-19.

Borredà, V. 1996. Pulmonados desnudos (Mollusca: Gastropoda: Pulmonata) del este de la Península Ibérica. Tesis Doctoral, Universitat de València, 475 pp. (inédita).

Britton, R.H. 1985. Life cycle and production of Hydrobia acuta Drap. (Gastropoda: Prosobranchia) in a hypersaline coastal lagoon. Hydrobiologia, 122, 219-230.

Brown, D. 1994. Freshwater Snails of Africa and their Medical Importante. $2^{\mathrm{a}}$ ed. Taylor \& Francis. London, 608 pp.

Burmeister, H. 1837. Handbuch der Naturgeschichte, vol. 2, Zoologie. Enslin, Berlin. i-xii, pp. 369-858.

Charpentier, J. de. 1837. Catalogue des mollusques terrestres et fluviatiles de la Suisse. Formant la seconde partie de la faune Helvétique. Nouveaux Mémoires de la Société Helvétique des Sciences Naturelles, 1, 1-28.

Cox, L.R. 1960. Thoughts on the classification of the Gastropoda. Proceedings of the Malacological Society of London, 33, 239-261.

Cuerda Barceló, J. 1987. Moluscos Marinos y Salobres del Pleistoceno Balear. Publicaciones de la Caja de Baleares "Sa Nostra". Palma de Mallorca, 418 pp.

Cuvier, G. 1795. Second Mémoire sur l'organisation et les rapports des animaux à sang blanc, dans lequel on traite de la structure des Mollusques et de leur division en ordre, lu à la société d'Histoire Naturelle de Paris, le 11 prairial an troisième [30 May 1795]. Magazin Encyclopédique, ou Journal des Sciences, des Lettres et des Arts, 1795 [1. année] 2, 433-449.

De Marfà, R. 2007. Microfauna del Pleistoceno inferior de Barranco León y Fuente Nueva 3 (Orce, Granada, España): estudio preliminar. Actas del III Encuentro de Jóvenes Investigadores en Paleontología, pp. 45-55.

De Renzi, M. 1978. La problemática de la fundamentación de la Paleoecología. Estudios Geológicos, 34, 559-570.

Delicado, D. 2013. Estudio de la diversidad del género Pseudamnicola y su radiación endémica en la región ibero-balear. taxonomía, filogenia y corología. Tesis Doctoral, Universidad Autónoma de Madrid, 291 pp. (inédita). http://hdl.handle.net/10261/134982.

Delicado, D., Machordom, A. \& Ramos, M.A. 2012. Underestimated diversity of Hydrobiid snails. The case of Pseudamnicola (Corrosella) (Mollusca: Caenogastropoda: Hydrobiidae). Journal of Natural History, 46, 25-89; doi: 10.1080/00222933.2011.623358.

Deshayes, G.P. 1855. Corbiculadae, 217-292. In: Catalogue of the Conchifera or Bivalve Shells in the Collection of the British Museum. Part 2, Petricoladae (concluded). British Museum, London.

Draparnaud, J.-P.-R. 1801. Tableau des Mollusques Terrestres et Fluviatiles de la France. Montpellier/Paris (Renaud/ Bossange, Masson \& Besson), 116 p; doi: 10.5962/bhl. title.40947.

Draparnaud, J.-P.-R. 1805. Histoire Naturelle des Mollusques Terrestres et Fluviatiles de la France. Paris (Levrault \& Schoell), 164 p; doi: 10.5962/bhl.title.12856.
Dubar, M. \& Magnin, F. 1992. Présence d'Helicelles (Helicellinae) dans le Pliocène du Midi de la France. Nouvelles données sur la dispersion du groupe au Pléistocene inférieur. Implications paléoclimatiques. Geobios, 25, 357-366.

Duval, M., Falguères, C., Bahain, J.J., Grün, R., Shao, Q., Aubert, M., Dolo, J.M., Agustí, J., Martínez-Navarro, B., Palmqvist, P. \& Toro-Moyano, I. 2012. On the limits of using combined U-series/ESR method to date fossil teeth from two Early Pleistocene archaeological sites of the Orce area (Guadix-Baza basin, Spain). Quaternary Research, 77, 482-491; doi: 10.1016/j.yqres.2012.01.003.

Ellis, A.E. 1926. British Snails, a Guide to the Non-Marine Gastropoda of Great Britain and Ireland, Pliocene to Recent. 275 pp. Clarendon Press, Oxford.

Erhmann, P. 1933. Mollusca. In: Die Tierwelt Mitteleuropas (eds Brohmer, P., Ehrmann, P. \& Ulmer, G.), 2, 264 pp.

Esu, D. \& Girotti, O. 1975. La malacofauna continentale del Plio-Pleistocene dell'Italia centrale. I. Paleontologia. Geologica Romana, 13, 203-294.

Esu, D. \& Girotti, O. 1991. Late Pliocene and Pleistocene assemblages of continental molluscs in Italy. A survey. II Quaternário, 4, 13-150.

Esu, D. \& Ghinassi, M. 2013. The non-marine molluses of the Plio-Pleistocene Upper Valdarno Basin (Tuscany, central Italy): depositional environments, palaeoecology and biochronology. Italian Journal of Geosciences, 132, 126-136; doi: 10.3301/IJG.2012.22.

Evagelopoulos, A., Spyrakos, E. \& Koutsoubas, D. 2009. Phytoplankton and macrofauna in the low salinity ponds of a productive solar saltworks: spatial variability of community structure and its major abiotic determinants. Global NEST Journal, 11, 64-72.

Evans, J.G. 1972. Land Snails in Archaeology. Seminar Press. London and New York, 436 pp.

Férussac, A.E.J.P.J.F. D’A. de. 1822. Tableaux Systématiques des Animaux Mollusques Classés en Familles Naturelles, dans lesquels on a Établi la Concordance de tous les Systèmes; Suivis d'un Prodrome Général pour tous les Mollusques Terrestres ou Fluviatiles, Vivants ou Fossiles. 110 pp. Bertrand, Sowerby, Paris, Londres.

Fetcher, R. \& Falkner, G. 1993. Moluscos. Ed. Blume. Barcelona, 287 pp.

Fitzinger, L.J. 1833. Systematisches Verzeichniss der im Erzherzogthume Oesterreich vorkommenden Weichthiere, als Prodrom einer Fauna desselben. Beiträge zur Landeskunde Oesterreich's unter der Enns, 3, 88-122.

Fleming, J. 1822. The Philosophy of Zoology, a General View of the Structure, Functions and Classification of Animals, 2. Constable \& Co., Edinburgh, 618 pp.

Fischer, P. 1856. Mélanges de conchyliologie. Actes de la Société Linnéenne de Bordeaux, 20, 357-400.

Fretter, V. \& Graham, A. 1978. The Prosobranch Mollusks of Britain and Denmark. Part 3. Neritacea, Viviparacea, Valvatacea, terrestrial and freshwater Littorinacea and 
Rissoacea. Journal of Molluscan Studies, Supplement, 5, 101-152.

García-Aguilar, J.M. \& Martín, J.M. 2000. Late Neogene to Recent continental history and evolution of de GuadixBaza basin (SE Spain). Revista de la Sociedad Geológica de España, 13, 65-77.

García-Aguilar, J.M. \& Palmqvist, P. 2011. A model of lacustrine sedimentation for the early Pleistocene deposits of Guadix-Baza basin (southeast Spain). Quaternary International, 243, 3-15; doi: 10.1016/j. quaint.2011.02.008.

García-Aguilar, J.M., Guerra-Merchán, A., Serrano, F., Palmqvist, P., Flores-Moya, A. \& Martínez-Navarro, B. 2014. Hydrothermal activity and its paleoecological implications in the latest Miocene to Middle Pleistocene lacustrine environments of the Baza Basin (Betic Cordillera, SE Spain). Quaternary Science Reviews, 96, 204-221; doi: 10.1016/j.quascirev.2013.07.009.

Gasull, L. 1974. Una interesante localidad con Melanoides tuberculata Müller en la provincia de Castellón de la Plana (Mollusc., Prosbr.). Boletín de la Sociedad de Historia Natural de Baleares, 19, 146-147.

Georgopoulou, E., Neubauer, T.A., Strona, G., Kroh, A., Mandic, O. \& Harzhauser, M. 2016. Beginning of a new age: How did freshwater gastropods respond to the Quaternary climate change in Europe? Quaternary Science Reviews, 149, 269- 278; doi: 10.1016/j. quascirev.2016.07.034.

Gill, T. 1871. Arrangement of the families of mollusks. Smithsonian Miscellaneous Collections, 227: xvi +49 pp.

Girod, A., Bianchi, I. \& Mariani, M. 1980. Gasteropodi, 1. Guide per il riconoscimento delle specie animali delle acque interne italiane, 7, 1-86.

Giusti, F., Manganelli, G. \& Bodon, M. 1998. A proposed neotype for Hydrobia acuta (Draparnaud, 1805). Journal of Conchology, 36, 1-8.

Gittenberger, E., Janssen, A.W., Kuijper, W.J., Kuiper, J.G.J., Meijer, T., Van Der Velde, G. \& De Vries, J.N. 1998. De Nederlandse Zoetwatermollusken. Recente en fossiele weekdieren uit zoet en brak water. Nederlandse Fauna, 2, 1-288.

Gliozzi, E., Abbazzi, L., Argenti, P., Azzaroli, A., Caloi, L., Capasso Barbato, L., Di Stefano, G., Esu, D., Ficcarelli, G., Girotti, O., Kotsaki, T., Masini, F., Mazza, P., Mezzabotta, C., Paombo, M.R., Petronio, C., Rook, L., Sala, B., Sardella, R., Zanalda, E. \& Torre, D. 1997. Biochronology of selected mammals, molluscs and ostracods from the Middle Pliocene to the Late Pleistocene in Italy. The state of the art. Rivista Italiana di Paleontologia e Stratigrafia, 103, 369-388.

Glöer, P. 2002. Die süsswassergastropoden Nord- und Mitteleuropas. Die Tierwelt Deutschlands, 73, 1-327.

Glöer, P. \& Meier-Brook, C. 1998. Süsswassermollusken. Ein Bestimmungsschlüssel für die Bundesrepublik Deuschland. 18 ed. Deutscher Jugendbund für Naturbeobbachtung, Hamburg, 136 pp.

Gofas, S., Moreno, D. \& Salas, C. (coords.). 2011. Moluscos Marinos de Andalucía. Volumen I, pp. i-xvi y 1-342;
Volumen II, pp. i-xii y 343-809. Servicio de Publicaciones e Intercambio Científico, Universidad de Málaga, Málaga.

Golikov, A.N. \& Starobogatov, Y. 1975. Systematics of Prosobranch gastropods. Malacologia, 15, 185-232.

Gray, J.E. 1840. Shells of molluscous animals. Synopsis of the contents of the British Museum, 42, 105-152.

Gray, J.E. 1857. Guide to the Systematic Distribution of Mollusca in the British Museum. Part I. Taylor \& Francis, London, xii $+230 \mathrm{pp}$.

Hartmann, J.D.W. 1821. System der Erd- und Flußschnecken der Schweiz. Mit vergleichender Aufzählung aller auch in den benachbarten Ländern, Deutschland, Frankreich und Italien sich vorfindenden Arten. In: Neue Alpina. Eine Schrift der Schweizerischen Naturgeschichte, Alpen- und Landwirthschaft gewiedmet (ed. Steinmüller, J.R.). Erster Band, 194-268, pl. 1-2.

Haszprunar, G. \& Huber, G. 1990. On the central nervous system of Smeagolidae and Rhodopidae, two families questionably allied with the Gymnomorpha (Gastropoda, Euthyneura). Journal of Zoology, 220, 185-199; doi: 10.1111/j.1469-7998.1990.tb04302.x.

Jaeckel, S.G.H. 1962. Ergänzungen und Berichtigungen zum rezenten und quartären Vorkommen der mitteleuropäischen Mollusken. Die Tierwelt Mitteleuropas, 2, 27-249.

Jeffreys, J.G. 1830. A synopsis of the testaceous pneumonobranchous Mollusca of Great Britain. Transactions of the Linnean Society of London, 16, 323-392.

Kabat, A.R. \& Hersler, R. 1993. The prosobranch Snail Family Hidrobiidae (Gastropoda: Rissoidea): review of clasification and supraespecific taxa. Smithsonian Contributions to Zoology, 547, 1-94.

Kerney, M.P. \& Cameron, R.A.D. 1999. Guide des Escargots et Limaces d'Europe. (Adaptation française. 372 pp. eds A. Bertrand Delachaux et Niestlé), Paris.

Kerney, M.P., Cameron, R.A.D. \& Jungbluth, J.H. 1983. Die Landschnecken Nord-und Mitteleuropas. Verlag Paul Parey. Hamburg und Berlin, 384 pp.

Kotsakis, T., Marcolini, F., De Rita, D., Conti, M. \& Esu, D. 2011.Three Late Pleistocene small mammal faunas from the Baccano maar (Rome, central Italy) Bollettino della Societa Paleontologica Italiana, 50, 103-110; doi: 10.4435/ BSPI.2011.11.

Koutsoubas, D., Arvanitidis, C., Dounas, C. \& Drummond, L. 2000. Community structure and dynamics of the Molluscan fauna in a Mediterranean lagoon (Gialova lagoon, SW Greece). Belgian Journal of Zoology, 130, 131-138.

Laviano, A. \& Penneta, L. 1980. Il deposito continentali plesitocenico di Fontana del Fico. Osservacioni, stratigrafiche e paleontologiche. Rivista Italiana di Paleontología e Stratigrafia, 86, 429-452.

Lemer, S., Bieler, R. \& Giribet, G. 2019. Resolving the relationships of clams and cockles: dense transcriptome sampling drastically improves the bivalve tree of life. Proceedings of the Royal Society B: Biological Sciences, 286, 1-9; doi: 10.1098/rspb.2018.2684.

Linnaeus, C. 1758. Systema Naturae per Regna Tria Naturae: Secundum Classes, Ordines, Genera, Species, cum Characteribus, Differentiis, Synonymis, Locis. Vol. 1. 
10th Edition. Impensis Direct. Laurentii Salvii, Holmiæ, Stockholm, 824 pp.

López, E., Aguilera, P.A., Schmitz, M.F., Castro, H. \& Pineda, F.D. 2010. Selection of ecological indicators for the conservation, management and monitoring of Mediterranean coastal salines. Environmental Monitoring and Assessment, 166, 241-56; doi: 10.1007/s10661-009-0998-2.

Lozek, V. 1964. Quartärmollusken der Tschechoslovakei, Praga, Akad. der Wissenschaften, 374 pp.

Martínez-Ortí, A. 1999. Moluscos terrestres testáceos de la Comunidad Valenciana. Tesis Doctoral. Universitat de València, $743 \mathrm{p}$ (inédita).

Medin, T., Martínez-Navarro, B., Rivals, F., MadurellMalapeira, J., Ros-Montoya, S., Espigares, M.P., Figueirido, B., Rook, L. \& Palmqvist, P. 2017. Late Villafranchian Ursus etruscus and other large carnivorans from the Orce sites (Guadix-Baza basin, Andalusia, southern Spain): Taxonomy, biochronology, paleobiology, and ecogeographical context. Quaternary International, 431, 20-41; doi: 10.1016/j.quaint.2015.10.053.

Meijer, T. 1990. Notes on Quaternary freshwater Mollusca of The Netherlands, with descriptions of some new species. Mededeligen ven de Werkgroep voor Tertiaire en Kwartaire Geologie, 26, 145-181.

Mitchell, A.J. \& Brandt, T.M. 2005. Temperature tolerance of red-rim Melania Melanoides tuberculatus, an exotic aquatic snail established in the United States. Transactions of the American Fisheries Society, 134, 126-131; doi: 10.1577/ FT03-178.1.

MolluscaBase. 2018. Accesible en http://www.molluscabase. org (10-02-2018).

Montfort, D. de. 1810. Conchyliologie Systématique, et Classification Méthodique des Coquilles; Offrant leurs Figures, leur Srrangement Générique, leurs Descriptions Caractéristiques, leurs Noms; ainsi que leur Synonymie en Plusieurs Langues. Ouvrage Destiné à Faciliter l'étude des Coquilles, ainsi que leur Disposition dans les Cabinets d'Histoire Naturelle. Coquilles Univalves, non Cloisonnées. 2. Schoell, Paris, 676 pp.

Mouthon, J. \& Kuiper, J.G.J. 1987. Inventaire des Sphaeriidae de France. Muséum National d'Histoire Naturelle. Inventaires de Faune et de Flore, 41, 1-60.

Müller, O.F. 1773-1774. Vermium terrestrium et fluviatilium, seu animalium infusoriorum, helminthicorum, et testaecorum, non marinorum, succincta historia. Havnice (Copenhagen) \& Lipsiæ (Leipzig), Heineck \& Faber, 1, 1-136; doi: 10.5962/bhl.title.462999.

Neumayr, M. 1883. Ueber einige tertiäre Süsswasserschnecken aus dem Orient. Neues Jahrbuch für Mineralogie, Geologie und Paläontologie, 1883, 37-44.

Økland, J. 1990. Lakes and Snails. Environment and Gastropoda in 1500 Norwegian Lakes. Universal Book Service, The Netherlands, $515 \mathrm{p}$.

Olivier, G.A. 1804. Voyage dans l'Empire Othoman, l'Égypte et la Perse, fair par Ordre du Gouvernement, Pendant les Six Premières Années de la République. Tome second, ii pp. +466 pp. + Errata (1 pp.), Atlas, 2d livraison: vii pp., pl. 18-32. Paris (H. Agasse).
Oms, O., Anadón, P., Agustí, J. \& Juliá, R. 2011. Geology and chronology of the continental Pleistocene archeological and paleontological sites of the Orce area (Baza basin, Spain). Quaternary International, 243, 33-43; doi: 10.1016/j.quaint.2011.03.048.

Pantazidou, A., Louvrou, I. \& Economou-Amilli, A. 2006. Euendolithic shell-boring cyanobacteria and chlorophytes from the saline lagoon Ahivadolimni on Milos Island, Greece. European Journal of Phycology, 41, 189-200; doi: 10.1080/09670260600649420.

Paulucci, M. 1878. Matériaux pour Servir à l'Étude de la Faune Malacologique Terrestre et Fluviatile de l'Italie et de ses Iles. Paris, Savy. 54 pp.

Pelseneer, P. 1935. Essai d'Ethologie Zoologique d'Après l'Etude de Mollusques. Académie Royale de Belgique. Classe des Sciences. Publications de la Fondation Agathon De Potter, 1, 1-662.

Pérès, J.M. \& Picard, J. 1964. Nouveau manuel de Bionomie benthique de la Mer Méditerranée. Recueil des Travaux de la Station Marine d'Endoume Bulletin, 31, 5-137.

Pérez-Quintero, J.C. 2007. Diversity, habitat use and conservation of freshwater molluses in the lower Guadiana River basin (SW Iberian Peninsula). Aquatic Conservation Marine and Freshwater Ecosystems, 17, 485-501; doi: 10.1002/aqc.796.

Pérez-Quintero, J.C. 2009. Moluscos dulceacuícolas de la Reserva de la Biosfera "Dehesas de Sierra Morena", SW de la Península Ibérica. Iberus, 27, 1-18.

Pérez-Quintero, J.C. 2011a. Distribution patterns of freshwater molluscs along environmental gradients in the southern Guadiana River basin (SW Iberian Peninsula). Hydrobiologia, 678, 65-76; doi: 10.1007/s10750-011-0821-2.

Pérez-Quintero, J.C. 2011b. Freshwater mollusc biodiversity and conservation in two stressed Mediterranean basins. Limnologica, 41, 201-212; doi: 10.1016/j. limno.2010.09.007.

Pérez-Quintero, J.C. 2012. Environmental determinants of freshwater mollusc biodiversity and identification of priority areas for conservation in Mediterranean water courses. Biodiversity and Conservation, 21, 3001-3016; doi: 10.1007/s10531-012-0351-x.

Pérez-Quintero, J.C. 2013. Mollusc communities along upstream-downstream gradients in small coastal basins of the south-western Iberian Peninsula. Hydrobiologia, 703, 165-175; doi: 10.1007/s10750-012-1356-x.

Pérez-Quintero, J.C., Bech, M. \& Huertas, J.L. 2004. Los moluscos de las aguas continentales de la provincia de Huelva (SO España). Iberus, 22, 19-31.

Petronio, C., Argenti, P., Caloi, L., Esu, D., Girotti, O. \& Sardella, R. 2000-2002. Updating Villafranchian mollusc and mammal faunas of Umbria and Latium (Central Italy). Geologica Romana, 36, 369-387.

Pfeiffer, C. 1821-1828. Naturgeschichte Deutscher Land-und Süsswasser-Mollusken. Weimar. Abt. 1: i-x, 1-134, pls 1-8 [1821]; Abt. 2: i-viii, 1-40, pls 1-8 [1825]; Abt. 3: i-vi, 1-84, pls 1-8 [1828].

Pfenninger, M., Cordellier, M. \& Streit, B. 2006. Comparing the efficacy of morphologic and DNA-based taxonomy in 
the freshwater gastropod genus Radix (Basommatophora, Pulmonata). BMC Evolutionary Biology, 6, 1-14; doi: 10.1186/1471-2148-6-100.

Pini, N. 1877. Molluschi terrestri e d'acque dolce viventi nel territorio d'Esino. Bullettino della Società Malacologica Italiana, 2, 67-205.

Plaziat, J.-C. \& Younis, W.R. 2005. The modern environments of molluscs in the southern Mesopotamia, Iraq: a guide to paleogeographical reconstructions of Quaternary fluvial, palustrine and marine deposits. Carnets de Géologiel Notebooks on Geology, Article 2005/01 (CG2005_A01); doi: 10.4267/2042/1453.

Pointier, J., Facon, B., Jarne, P. \& David, P. 2003. Les thiaridés, des gasteropodes envahisseurs des eaux douces tropicales. Xenophora, 104, 14-20.

Poli, J.X. 1791-1795. Testacea Utriusque Siciliae Eorumque Historia et Anatome Tabulis Aeneis Illustrata. Ex Regio Typographeio, Parmae. Vol. 1: pp. i-lxxiii, [1-6], i-x, 1-90, 1-50, [1], 1-74, pls 1-18 [1791]; Vol. 2 [1795]: pp. i-xlix, [1-2], 75-264, pls 19-39.

Radoman, P. 1969. On the Taxonomy and Biogeography of Hydrobiidae. Malacologia, 9, 173-177.

Radoman, P. 1973. New classification of fresh and brackish water Prosobranchia from the Balkans and Asia Minor. Posebno lzdanie Prirodonaucen Muzej na Makedonija, 32, 1-30.

Radoman, P. 1977. Hydrobiidae auf der Balkanhalbinsel und in Kleinasien. Archiv für Molluskenkunde, 107, 203-223.

Rafinesque, C.S. 1815. Analyse de la Nature ou Tableau de l'Univers et des Corps Organisés. 223 p. Privately published by author, Palermo.

Robles, F. 1989. Moluscos continentales del Plio-Pleistoceno de la cuenca de Guadix-Baza. In: Geología y Paleontología de la Cuenca de Guadix-Baza (eds. Alberdi, M.T. \& Bonadona, F.P.). Trabajos sobre Neógeno-Cuaternario, 11, 127-138.

Rodríguez-Lázaro, J., Robles, F., Martín, M., Anadón, P., Utrilla, R. \& Vázquez, A. 1997. La sucesión lacustre pliocena de Villarroya (La Rioja). Principales características paleoambientales basadas en ostrácodos y moluscos. Geogaceta, 22, 185-188.

Roessler, M.A., Beardsley, G.L. \& Tabb, D.C. 1977. New records of the introduced snail, Melanoides tuberculata (Mollusca: Thiaridae) in south Florida. Florida Scientist, 40, 87-94.

Rosso, J.C. 1983. Paléofaunes. Mollusques. In: Sahara ou Sahel? (eds. Petit-Maire, N. \& Riser, P.). Lamy, Marseille, pp. 157-171.

Royo Gómez, J. 1922. El Mioceno Continental Ibérico y su Fauna Malacológica. Comisión de Investigaciones Paleontológicas y Prehistóricas. Junta de Ampliación de Estudios e Investigaciones Científicas, Memorias, 30, 1-230.

Royo Gómez, J. 1928. El Terciario continental de Cuenca Alta del Tajo. Memorias del Instituto Geológico y Minero de España, 1-89.

Schlickum, W.R. \& Puisségur, J.J. 1977. Die Molluskenfauna des Altpleistozäns von St. Bernard (Département Côted'Or). Archiv für Molluskenkunde, 107, 273-283.
Schlickum, W.R. \& Puisségur, J.J. 1978. Die Molluskenfauna der schichten mit Viviparus burgundinus und Pyrgula nodotiana von Montagny-les-Beaune (Département Côte d'Or). Archiv für Molluskenkunde, 109, 1-26.

Schmidt, A. 1855. Der Geschlechtsapparat der Stylommatophoren in taxonomischer Hinsicht. Abhandlungen des naturwissenschaftlichen Vereins für Sachsen und Thüringen in Halle, 1, 1-52.

Schniebs, K., Glöer, P., Vinarski, M.V. \& Hundsdoerfer, A.K. 2011. Intraspecific morphological and genetic variability in Radix balthica (Linnaeus, 1758) (Gastropoda: Basommatophora: Lymnaeidae) with morphological comparison to other European Radix species. Journal of Conchology, 40, 657-678.

Schniebs, K., Glöer, P., Vinarski, M.V. \& Hundsdörfer, A.K. 2013. Intraspecific morhological and genetic variability in the European freshwater snail Radix labiata (Rossmaessler, 1835) (Gastropoda: Basommatophora: Lymnaeidae). Contributions to Zoology, 82, 55-68.

Soler, J., Moreno, D., Araujo, R. \& Ramos, M.A. 2006. Diversidad y distribución de los moluscos de agua dulce en la Comunidad de Madrid (España). Graellsia, 62 (número extraordinario), 201-252.

Stimpson, W. 1865. Researches upon the Hydrobiinae and allied forms: chiefly made from materials in the Museum of the Smithsonian Institution. Smithsonian Miscellaneous Collections, 7, 1-59.

Studer, S. 1820. Kurzes Verzeichniss der bis jetzt in unserm Vaterlande entdeckten Conchylien. Naturwissenschaftlicher Anzeiger der Allgemeinen Schweizerischen Gesellschaft für die Gesammten Naturwissenschaften, 3, 83-90, 91-94.

Toro-Moyano, I., Martínez-Navarro, B., Agustí, J., Souday, C., Bermúdez de Castro, J.M., Martinón-Torres, M., Fajardo, B., Duval, M., Falguères, C., Oms, O., Parés, J.M., Anadón, P., Julià, R., García-Aguilar, J.M., Moigne, A.M., Espigares, M.P., Ros-Montoya, S. \& Palmqvist, P. 2013. The oldest human fossil in Europe, from Orce (Spain). Journal of Human Evolution, 65 1-9; doi: doi. org/10.1016/j.jhevol.2013.01.012.

Turton, W. 1831. A Manual of the Land and Freshwater Shells of the British Islands Arranged According to the more Modern Systems of Classification; and Described from Perfect Specimens in the Author's Cabinet: with Coloured Plates of every Species. 152 pp. Longman, Orme, Brown, Green\&Longmans, London.

Truc, G. 1971. Gastéropodes continentaux néogènes du Bassin rhodanien. Document du Laboratoire Géologique de la Université de Lyon, $11 \mathrm{~S}, 79-129$.

Vera, J.A. 1970. Estudio estratigráfico de la depresión de Guadix-Baza. Boletín Geológico y Minero, 81, 429-462.

Wenz, W. 1923-1930. Gastropoda Extramarina Tertiaria. Fossilium Catalogus, I.- Animalia, pars 17, 18, 20-23, 32, 38, 40, 43 y 46. Berlín, 3387 pp.

Wilke, T. \& Pfenninger, M. 2002. Separating historic events from recurrent processes in cryptic species: phylogeography of mud snails (Hydrobia spp.). Molecular Ecology, 11, 1439-1451; doi: 10.1046/j.1365294X.2002.01541.x. 
Wilke, T., Rolán, E. \& Davis, G.M. 2000. The mudsnail genus Hydrobia ss in the northern Atlantic and western Mediterranean: a phylogenetic hypothesis. Marine Biology, 137, 827-833; doi: 10.1007/s002270000407.

Wilke, T., Davis, G.M., Falniowski, A., Giusti, F., Bodon, M. \& Szarowska, M. 2001. Molecular systematics of
Hydrobiidae (Mollusca: Gastropoda: Rissooidea): testing monophyly and phylogenetic relationships. Proceedings of the Academy of Natural Sciences of Philadelphia, 151, 1-21; doi: 10.1635/0097-3157(2001)151[0001:MSOHM $\mathrm{G}] 2.0 . \mathrm{CO} ; 2$. 
